\title{
Testing for Structural Breaks in Time Series Regressions with Heavy-tailed Disturbances*
}

\author{
Stefan Mittnik \\ Institute of Statistics and Econometrics \\ University of Kiel \\ Olshausenstr. 40, D-24098 Kiel, Germany \\ Svetlozar T. Rachev \\ Department of Statistics and Applied Probability \\ University of California \\ Santa Barbara, CA 93106-3110, U.S.A. \\ Gennady Samorodnitsky \\ School of Operations Research \& Industrial Engineering \\ Cornell University \\ Ithaca, NY 14853-3801, U.S.A.
}

October 1998

\begin{abstract}
We investigate the asymptotic behavior of the OLS residual-based CUSUM test for parameter constancy in a dynamic regression with heavy-tailed disturbances. We extend previous results by relaxing the finite-variance assumption and consider disturbances in the domain of attraction of a stable Paretian law. The main result is a functional limit theorem for the self-normalizing CUSUMs of OLS residuals. We report on a simulation study of the resulting prelimiting and limiting processes. Finally, we provide response-surface approximations of critical values for the CUSUM test statistic.
\end{abstract}

KEYwords: CUSUM test, least squares residuals, infinite variance, stable Paretian, response-surface technique.

${ }^{*}$ The research was partly conducted while S. Rachev visited the University of Kiel with support from the Alexander-von-Humboldt Foundation for U.S. Scientists. The research of S. Mittnik was supported by the Deutsche Forschungsgemeinschaft and that of G. Samorodnitsky by NSF grants DMS-97-04982 and DMI-97-13549 as well as by the NSA grant MDA904-98-1-0041 at Cornell University. 


\section{Introduction}

CUSUM-type tests of the stability over time of coefficient vector $\beta$ in the dynamic linear regression $Y_{i}=X_{i}^{\prime} \beta+U_{i}$ are commonly used in the econometric work. Brown, Durbin and Evans (1975) proposed CUSUM tests based on recursive residuals; MacNeill (1978) considered a CUSUM test using ordinary least squares (OLS) residuals; while McCabe and Harrison (1980) studied CUSUM-of-squares tests based on the assumption of independent, identically distributed (i.i.d.) disturbances. Ploberger and Krämer (1992) (hereafter, PK) provided a functional limit theorem for the sums of OLS residuals,

$$
U_{i}^{(n)}=Y_{i}-X_{i}^{\prime} \hat{\beta}^{(n)}, \quad 1 \leq i \leq n
$$

where $\hat{\beta}^{(n)}$ denotes the OLS estimator for $\beta$. Their main result describes a set of regularity conditions, which imply that the cumulative process $\sum_{i=1}^{[n t]} U_{i}^{(n)}, 0 \leq t \leq 1,-$ after applying the for finite-variance processes usual normalization - converges weakly to a Brownian bridge. ${ }^{1}$

Since the influential work of Mandelbrot (1963) and Fama (1965) there has been substantial empirical evidence that data arising in speculative markets tend to have distributions that are fat-tailed and excessively peaked around the center. This makes-as suggested by Mandelbrot and Fama - the stable Paretian (in short, $\alpha$-stable) distribution a much more realistic model than the Gaussian one. ${ }^{2}$ The $\alpha$-stable family includes the Gaussian distribution as the special case, which is obtained when the stable index or tail-thickness parameter equals two; but it also accommodates the infinite-variance assumption when $0<\alpha<2$. Accepting the $\alpha$-stable hypothesis, it is reasonable to allow the disturbances in a regression, which involves asset returns as dependent variables, to have infinite variance.

The CUSUM squares test under the infinite-variance assumption was first studied in Loretan and Phillips (1994). Kim, Mittnik and Rachev (1996) (hereafter, KMR)

\footnotetext{
${ }^{1}$ Ploberger and Krämer (1992) modified the CUSUM test to allow for correlated and heteroskedastic OLS residuals.

${ }^{2}$ For discussions of $\alpha$-stable distributions in modeling asset returns we refer to Mittnik and Rachev (1993), McCulloch (1996), Mittnik, Rachev and Paolella (1997) and Mittnik and Rachev (1998).
} 
replaced the Gaussian assumption in PK's functional limit theorem by the assumption of non-Gaussian stable disturbances. More specifically, KMR modified the standard test statistic by replacing the sample standard deviation by the sample $p$ th norm, $0<p<\alpha$, and showed that, after a proper normalization, the CUSUMs of OLSresiduals converge to a Lévy bridge (see also Rachev, Kim and Mittnik (1997)). The limiting procedure enables us to test for the constancy of the regression coefficient $\beta$ by constructing confidence regions based on the Lévy bridge.

The drawback of the approach in KMR is that the resulting test statistic depends on the unknown stable index $\alpha$. Moreover, in order to use consistently a sample estimate of the theoretical $p$ th moment one has to assume that the disturbances themselves are $\alpha$-stable. In this paper we avoid these drawbacks. We provide a functional limit theorem for a self-normalizing version of the CUSUM test put forth in PK. In doing so, we only assume that disturbances are in the domain of attraction of an $\alpha$-stable law. The resulting test statistic does not depend on the unknown stable index $\alpha$ (of course, its limiting distribution does depend on that index.) It turns out that the limiting results deviate substantially from those in PK, once we allow disturbances to be heavy-tailed.

The paper is organized as follows. Section 2 establishes some notation and summarizes relevant facts about $\alpha$-stable distributions and their domains of attraction. In Section 3 we derive our main result, the functional limit theorem for the self-normalizing OLS-residual process arising in the CUSUM test when disturbances are in the domain of normal attraction of an $\alpha$-stable law. Simulation results on the prelimiting and limiting processes are presented in Section 4. There, we also present a set of critical values, which can easily be implemented in applied work. Section 5 concludes. An appendix contains the proofs of various technical statements used in Section 3.

\section{Stable Laws and Their Domains of Attraction.}

There are several ways of defining an $\alpha$-stable distribution (see Zolotarev, 1986; Samorodnitsky and Taqqu, 1994, and the references therein). The classical definition, given 
in Lévy (1937), states that a random variable (r.v.) $X$ is stable, if for any positive numbers $A$ and $B$ there is a positive number, $C$, and a real number, $D$, such that $A X_{1}+B X_{2} \stackrel{d}{=} C X+D$, where $X_{1}$ and $X_{2}$ are independent r.v.'s with $X_{i} \stackrel{d}{=} X, i=1,2$ and " $=$ " denotes equality in distribution. For any stable r.v. $X$ there is a number $\alpha \in(0,2]$ such that $C$ satisfies $C^{\alpha}=A^{\alpha}+B^{\alpha}$ (see Feller, 1971, Sec. 17.4). The exponent $\alpha$ is called the index of stability. For $\alpha<2$ a non-degenerate stable r.v. $X$ with index of stability $\alpha$ satisfies $P(|X|>t) \sim c t^{-\alpha}$ for some $c>0$ as $t \rightarrow \infty$, and the left and right tails of $X$ are balanced as in (4) below. Hence, if $\alpha<2$, the tails of the distribution of a stable r.v. are fatter than those of the normal distribution; and the tail-thickness increases as $\alpha$ decreases. This is why $\alpha$ is also referred to as the tail-thickness parameter. If $\alpha<2$, moments of order $\alpha$ or higher do not exist. A stable r.v. with index $\alpha$ is said to be $\alpha$-stable. A Gaussian random variable is a 2 -stable random variable (i.e., $\alpha=2$ ). Indeed, if $X_{1}$ and $X_{2}$ are independent normal with a common mean $\mu$ and variance $\sigma^{2}$, then $A X_{1}+B X_{2} \sim N\left((A+B) \mu,\left(A^{2}+B^{2}\right) \sigma^{2}\right)$; i.e., we have $C=\left(A^{2}+B^{2}\right)^{\frac{1}{2}}$ and $D=(A+B-C) \mu$.

Closed-form expressions of $\alpha$-stable distributions or their densities exist only in few special cases. However, the logarithm of the characteristic function (ch.f.), $f(\theta)=$ $E e^{i \theta X}$, of $\alpha$-stable r.v. $X$, can be written as

$$
\ln f(\theta)= \begin{cases}-\sigma^{\alpha}|\theta|^{\alpha}\left[1-i \beta \operatorname{sign}(\theta) \tan \frac{\pi \alpha}{2}\right]+i \mu \theta, & \text { for } \alpha \neq 1, \\ -\sigma|\theta|\left[1+i \beta \frac{\pi}{2} \operatorname{sign}(\theta) \ln |\theta|\right]+i \mu \theta, & \text { for } \alpha=1\end{cases}
$$

$\theta \in \mathbf{R}$, where $\mu \in \mathbf{R}$ is the location parameter; $\sigma \geq 0$ is the scale parameter; and $\beta \in[-1,1]$ is the skewness parameter. The distribution function of an $\alpha$-stable r.v. satisfying (1) is denoted by $S(x ; \alpha, \beta, \sigma, \mu)$. If $\beta=0$, the distribution is symmetric. The location parameter shifts the distribution to the left or right, while the scale parameter expands or contracts it about $\mu$. If $X$ has ch.f. (1) we write $X \stackrel{d}{=} S_{\alpha}(\beta, \sigma, \mu)$. For $\alpha=2, S_{2}(\beta, \sigma, \mu)$ is the normal distribution $N\left(\mu, 2 \sigma^{2}\right)$. Unless both $\alpha=1$ and $\beta \neq 0$ the standardized version $(X-\mu) / \sigma$ of $X \stackrel{d}{=} S_{\alpha}(\beta, \sigma, \mu)$ has distribution $S_{\alpha}(\beta, 1,0)$.

A sample $U_{1}, U_{2}, \ldots$ of i.i.d. observations is said to be in the domain of attraction 
of an $\alpha$-stable law with index $\alpha \in(0,2]$ if there exist constants $a_{n} \geq 0$ and $b_{n} \in \mathbf{R}$ such that

$$
a_{n}^{-1} S_{n}-b_{n} \stackrel{w}{\Rightarrow} X
$$

where $S_{n}=U_{1}+\cdots+U_{n}, X$ is a non-degenerate $\alpha$-stable r.v., and " $\stackrel{w}{\Rightarrow}$ " stands for weak convergence. In particular, when $U_{i}^{\prime}$ 's are $\alpha$-stable, $U_{1} \stackrel{d}{=} S_{\alpha}(\beta, \sigma, \mu),(2)$ holds and, moreover, we have $a_{n}^{-1} S_{n}-b_{n} \stackrel{d}{=} U_{1}$, with $a_{n}=n^{1 / \alpha}$ and $b_{n}=\mu\left(n^{1-1 / \alpha}-1\right)$ for $\alpha \neq 1$, and $b_{n}=\frac{2}{\pi} \sigma \beta n \ln n$ for $\alpha=1$.

The assumption that the disturbances $U_{i}$ 's are in the domain of attraction of an $\alpha$-stable law is a relaxation of the assumption of $\alpha$-stable distributed disturbances. In fact, for $\alpha<2$ the domain-of-attraction condition (2) is equivalent to the assumption that the tail behavior of $U_{i}$ is of the Pareto-Lévy form (cf. Feller, 1966, p. 303):

$$
P\left(\left|U_{i}\right|>t\right)=t^{-\alpha} L(t), \quad t>0,
$$

where $L(t)$ is a slowly varying function as $t \rightarrow \infty,{ }^{3}$ and

$$
\lim _{t \rightarrow \infty} \frac{P\left(U_{i}>t\right)}{P\left(\left|U_{i}\right|<t\right)}=p, \quad \lim _{t \rightarrow \infty} \frac{P\left(U_{i}<-t\right)}{P\left(\left|U_{i}\right|<t\right)}=q
$$

for some $p \geq 0$ and $q \geq 0$ with $p+q=1$.

We shall further assume that $U_{i}$ are in the domain of normal attraction of an $\alpha-$ stable law, that is, for some $c>0$,

$$
P\left(\left|U_{i}\right|>t\right) \sim c t^{-\alpha} \text { as } t \rightarrow \infty
$$

and furthermore the limiting relationships (4) hold. ${ }^{4}$

\footnotetext{
${ }^{3} L(t)$ is a slowly varying function as $t \rightarrow \infty$, if for every constant $c>0$, $\lim _{t \rightarrow \infty} L(c t) / L(t)$ exists and is equal to 1 . We will use $L$ or $l$ to denote a slowly varying function.

${ }^{4}$ The $U_{i}$ 's are in the domain of normal attraction of an $\alpha$-stable law, if (2) holds with $a_{n}=c_{0} n^{1 / \alpha}$ for some positive constant $c_{0}$. Note that when the $U_{i}$ 's are in the general domain of attraction, then, in (2), $a_{n}=n^{1 / \alpha} L(n)$ for some slowly varying function $L(n)$ as $n \rightarrow \infty$.
} 
Let $g(x)=1 / P\left(\left|U_{i}\right|>x\right)$ and consider the generalized inverse of $g(x)$ :

$$
g^{\leftarrow}(y):=\sup \{x: g(x) \leq y\}
$$

Set

$$
a_{n}:=g^{\leftarrow}(n), n \geq 1
$$

then, as $n \rightarrow \infty, a_{n} \sim c n^{1 / \alpha}$.

Next, we need some basic definitions and results on Poisson random measures (see Resnick (1987)). Let $E$ be a locally compact topological space with a countable base and let $\mathcal{E}$ be the Borel $\sigma$-algebra of subsets of $E$. A point measure $m$ on $\mathcal{E}$ with support $\left\{x_{i}, i \geq 1\right\} \subset E$ is defined by

$$
m=\sum_{i=1}^{\infty} \epsilon_{x_{i}}
$$

where

$$
\epsilon_{x_{i}}(A)=\left\{\begin{array}{ll}
1, & \text { if } x_{i} \in A, \\
0, & \text { if } x_{i} \notin A,
\end{array} \quad A \in \mathcal{E} .\right.
$$

A point process $N$ on $E$ is a random element,

$$
N:(\Omega, \mathcal{A}, P) \rightarrow\left(M_{P}(E), \mathcal{M}_{p}(E)\right)
$$

on the original probability space $(\Omega, \mathcal{A}, P)$ with values in the space $M_{P}(E)$ of all point measures on $E$ with the $\sigma$-algebra $\mathcal{M}_{P}(E)$ generated by the sets $\left\{m \in M_{P}(E): m(F) \in\right.$ $B\}, F \in \mathcal{E}$, and $B$ a Borel set in $[0, \infty]$, i.e., $B \in \mathcal{B}([0, \infty])$.

Let $\mu$ be a Radon measure on $(E, \mathcal{E})$, that is, $\mu$ is finite on all compact subsets of E. A point process $N$ is called Poisson random measure (PRM) with mean measure $\mu$, if

\footnotetext{
${ }^{5}$ Here, and in what follows, $c$ stands for a generic constant, which can be different in various contexts.
} 
(i) for every $F \in \mathcal{E}$, and every $k \in \mathbf{N}:=\{1,2, \ldots\}$,

$$
P(N(F)=k)= \begin{cases}\frac{\mu(F)^{k}}{k !} e^{-\mu(F)}, & \text { if } \mu(F)<\infty \\ 0, & \text { if } \mu(F)=\infty\end{cases}
$$

and if

(ii) $F_{1}, \ldots, F_{k}$ (for every $k \in \mathbf{N}$ ) are mutually disjoint sets in $\mathcal{E}$, then $N\left(F_{1}\right), \ldots$, $N\left(F_{k}\right)$ are independent r.v.

Consider next an array of r.v.'s $\left(U_{n, j}, j \geq 1, n \geq 1\right)$ with values in $(E, \mathcal{E})$ and assume that for each $n\left(U_{n, j}\right)_{j \geq 1}$ are i.i.d. r.v.'s. Suppose that the sequence of finite measures defined by

$$
\mu_{n}(A):=n P\left(U_{n, 1} \in A\right), A \in \mathcal{E},
$$

converges vaguely to a Radon measure $\mu$ on $(E, \mathcal{E}){ }^{6}$

Proposition 1. (see Resnick (1987), Proposition 3.21). Let

$$
\xi_{n}=\sum_{k \geq 1} \varepsilon_{\left(\frac{k}{n}, U_{k, n}\right)}
$$

and $\xi$ be a PRM on $[0, \infty) \times E$ with mean measure $d t \times d \mu$. Then

$$
\mu_{n} \stackrel{v}{\rightarrow} \mu
$$

if and only if ${ }^{7}$

$$
\xi_{n} \stackrel{щ}{\Rightarrow} \xi
$$

We now apply the above proposition to the sequence $\left(U_{i}\right)_{i \geq 1}$ of i.i.d. r.v.'s in the domain of normal attraction of an $\alpha$-stable law. Namely, we take $E:=[-\infty, \infty] \backslash$ $\{(0)\}$ ), (i.e., relatively compact sets are those bounded away from the origin) and set

\footnotetext{
${ }^{6}\left(\mu_{n}\right)_{n \geq 1}$ converges vaguely to $\mu\left(\mu_{n} \stackrel{v}{\rightarrow} \mu\right)$ if $\limsup _{n \rightarrow \infty} \mu_{n}(K) \leq \mu(K)$ for all compact sets $K \subset E$ and $\liminf _{n \rightarrow \infty} \mu_{n}(G) \geq \mu(G)$ for all open relatively compact sets $G \subset E$.

$\stackrel{7}{\Rightarrow}$ in (11) stands for the weak convergence of stochastic point processes, in this case the weak convergence in the space $M_{P}([0, \infty) \times E)$.
} 
in Proposition $1, U_{k, n}=\frac{U_{k}}{a_{n}}$, where $a_{n}$ was defined as $g^{\leftarrow}(n)$, see (6). Then, as $n \rightarrow \infty$,

$$
\mathbf{X}_{n}^{*}:=\sum_{k=1}^{\infty} \varepsilon_{\left(\frac{k}{n}, \frac{U_{k}}{a_{n}}\right)} \stackrel{щ}{\Rightarrow} \sum_{i} \varepsilon_{\left(t_{i}, j_{i}\right)}=: \mathbf{X}^{*}
$$

in $M_{p}([0, \infty) \times E)$, where the limit in $(12)$ is a PRM with mean measure $d t \times d \nu$, and

$$
\nu(d x)=\alpha p x^{-(1+\alpha)} d x \mathbf{1}(\{x>0\})+\alpha q|x|^{-(1+\alpha)} d x \mathbf{1}(\{x>0\})
$$

(see Formula (4.70) in Resnick (1987), p. 226). Furthermore, the points of $\mathbf{X}^{*}$ on $\{t \leq 1\}$ arranged in the non-increasing order by the magnitude of the "jumps" $j_{i}$ can be represented in distribution as

$$
\left(U_{j}^{0} \delta_{j}, \Gamma_{j}^{-1 / \alpha}\right)_{j \geq 1}
$$

where $\left(U_{j}^{0}\right)_{j \geq 1},\left(\delta_{j}\right)_{j \geq 1}$ and $\left(\Gamma_{j}\right)_{j \geq 1}$ are three independent sequences of r.v.'s; $\left(U_{j}^{0}\right)_{j \geq 1}$ are i.i.d. r.v.'s uniformly distributed on $[0,1] ;\left(\delta_{j}\right)_{j \geq 1}$ are i.i.d. random signs, $P\left(\delta_{j}=1\right)=$ $1-P\left(\delta_{j}=-1\right)=p$; and $\left(\Gamma_{j}\right)_{j \geq 1}$ are the standard Poisson arrivals, i.e., $\Gamma_{j}=e_{1}+\ldots+e_{j}$, where $\left(e_{j}\right)_{j \geq 1}$ is a sequence of i.i.d. exponential r.v.'s with mean 1.

\section{CUSUM Test and Its Limiting Distribution}

Consider the regression model

$$
Y_{i}=X_{i}^{\prime} \beta+U_{i}, \quad 1 \leq i \leq n,
$$

where $\beta=\left(\beta_{0}, \beta_{1}\right)^{\prime}$,

$$
X_{i}=\left(\begin{array}{c}
1 \\
Z_{i}
\end{array}\right), 1 \leq i \leq n,
$$

with

$$
\frac{1}{n} \sum_{i=1}^{n} Z_{i} \rightarrow_{n \rightarrow \infty} 0
$$




$$
\frac{1}{n} \sum_{i=1}^{n} Z_{i}^{2} \rightarrow_{n \rightarrow \infty} R>0
$$

and $U_{1}, U_{2}, \ldots$ are i.i.d. r.v.'s in the domain of normal attraction of an $\alpha$-stable law. Define the normalizing constants $a_{n}$ by $(6)$, and so, $a_{n} \sim c n^{1 / \alpha}$ as $n \rightarrow \infty$. In addition, we assume:

$$
\begin{aligned}
& \text { (A1) If } 1<\alpha<2 \text {, then } E\left(U_{1}\right)=0 . \\
& \text { (A2) If } \alpha=1 \text {, then } \int_{-n}^{n} x d F_{U_{1}}(x) \rightarrow_{n \rightarrow \infty} 0,
\end{aligned}
$$

where $F_{U_{1}}(x)$ is the distribution function of $U_{1} \cdot{ }^{8}$ No additional assumptions are imposed for the case $0<\alpha<1$.

The OLS estimator for $\beta$ and the OLS residuals are given by

$$
\hat{\beta}^{(n)}:=\left(\sum_{i=1}^{n} X_{i} X_{i}^{\prime}\right)^{-1} \sum_{j=1}^{n} Y_{j} X_{j}
$$

and

$$
U_{i}^{(n)}:=Y_{i}-X_{i}^{\prime} \hat{\beta}^{(n)}, 1 \leq i \leq n
$$

respectively.

Our main result is Theorem 1 below; it provides a functional limit theorem for the CUSUM process based on self-normalized OLS residuals. We shall examine the weak limit in the Skorohod space $D[0,1]$ of the following sequence of processes: for $\xi \in[0,1]$ and $n \geq 1$ let $^{9}$

$$
X_{n}(\xi):=\frac{\sum_{i=1}^{[n \xi]} U_{i}^{(n)}}{\left(\sum_{i=1}^{n}\left(U_{i}^{(n)}\right)^{2}\right)^{1 / 2}}=\frac{a_{n}^{-1} \sum_{i=1}^{[n \xi \xi} U_{i}^{(n)}}{\left(a_{n}^{-2} \sum_{i=1}^{n}\left(U_{i}^{(n)}\right)^{2}\right)^{1 / 2}}
$$

Next, let

$$
X_{\infty}(\xi):=\frac{\sum_{j=1}^{\infty} \delta_{j} \Gamma_{j}^{-1 / \alpha}\left(\mathbf{1}_{\left\{V_{j} \leq \xi\right\}}-\xi\right)}{\left(\sum_{j=1}^{\infty} \Gamma_{j}^{-2 / \alpha}\right)^{1 / 2}}, 0 \leq \xi \leq 1
$$

\footnotetext{
${ }^{8}$ Note that Assumption A2 implies, in particular, that $U_{1}, U_{2}, \ldots$ are attracted to a symmetric 1 -stable (Cauchy) law. No symmetry assumptions are made in the case $\alpha \neq 1$.

${ }^{9}$ Notation $[a]$ denotes the integer part of $a$.
} 
where the sequences $\left(V_{j}\right)_{j \geq 1},\left(\delta_{j}\right)_{j \geq 1}$ and $\left(\Gamma_{j}\right)_{j \geq 1}$ are independent. $\left(V_{j}\right)_{j \geq 1}$ are "random signs" (that is, i.i.d. r.v.'s) with uniformly distributed on $[0,1], V_{j} \stackrel{d}{=} U(0,1) ;\left(\delta_{j}\right)_{j \geq 1}$ are "random signs" with $P\left(\delta_{j}=1\right)=1-P\left(\delta_{j}=-1\right)=p$, where $p$ is defined in (4); and $\left(\Gamma_{j}\right)_{j \geq 1}$ are the arrivals of a standard Poisson process.

Theorem 1. Under assumption $\mathbf{A 1}$, if $1<\alpha<2$, or under assumption $\mathbf{A 2}$, if $\alpha=1$, the sequence of processes $\left(X_{n}(\xi)\right)_{0 \leq \xi \leq 1}$ converges weakly in the Skorohod $J_{1}$ topology in $D[0,1]$ to the process $\left(X_{\infty}(\xi)\right)_{0 \leq \xi \leq 1}$ :

$$
\mathbf{X}_{n} \stackrel{⿱}{\Rightarrow} \mathbf{X}_{\infty}
$$

Proof. Observe that the OLS estimator $\hat{\beta}^{(n)}=\left(\hat{\beta}_{0}^{(n)}, \hat{\beta}_{1}^{(n)}\right)^{\prime}$ has the form

$$
\begin{aligned}
\left(\begin{array}{c}
\hat{\beta}_{0}^{(n)} \\
\hat{\beta}_{1}^{(n)}
\end{array}\right)= & \left(\begin{array}{cc}
n & \sum_{i=1}^{n} Z_{i} \\
\sum_{i=1}^{n} Z_{i} & \sum_{i=1}^{n} Z_{i}^{2}
\end{array}\right)^{-1}\left(\begin{array}{c}
\sum_{j=1}^{n}\left(\beta_{0}+\beta_{1} Z_{j}+U_{j}\right) \\
\sum_{j=1}^{n}\left(\beta_{0} Z_{j}+\beta_{1} Z_{j}^{2}+U_{j} Z_{j}\right)
\end{array}\right) \\
= & \frac{1}{n \sum_{i=1}^{n} Z_{i}^{2}-\left(\sum_{i=1}^{n} Z_{i}\right)^{2}}\left(\begin{array}{cc}
\sum_{i=1}^{n} Z_{i}^{2} & -\sum_{i=1}^{n} Z_{i} \\
-\sum_{i=1}^{n} Z_{i} & n
\end{array}\right) \\
& \times\left(\begin{array}{c}
\sum_{j=1}^{n}\left(\beta_{0}+\beta_{1} Z_{j}+U_{j}\right) \\
\sum_{j=1}^{n}\left(\beta_{0} Z_{j}+\beta_{1} Z_{j}^{2}+U_{j} Z_{j}\right)
\end{array}\right) .
\end{aligned}
$$

Therefore,

$$
\hat{\beta}_{0}^{(n)}=\beta_{0}+\frac{\sum_{i=1}^{n} Z_{i}^{2} \sum_{j=1}^{n} U_{j}-\sum_{i=1}^{n} Z_{i} \sum_{j=1}^{n} U_{j} Z_{j}}{n \sum_{i=1}^{n} Z_{i}^{2}-\left(\sum_{i=1}^{n} Z_{i}\right)^{2}},
$$

and

$$
\hat{\beta}_{1}^{(n)}=\beta_{1}+\frac{n \sum_{i=1}^{n} U_{i} Z_{i}-\sum_{i=1}^{n} U_{i} \sum_{j=1}^{n} Z_{j}}{n \sum_{i=1}^{n} Z_{i}^{2}-\left(\sum_{i=1}^{n} Z_{i}\right)^{2}} .
$$

From (27) and (28), we conclude

$$
\begin{aligned}
U_{i}^{(n)} & =\beta_{0}+\beta_{1} Z_{i}+U_{i}-\left(1, Z_{i}\right)\left(\hat{\beta}_{0}^{(n)}, \hat{\beta}_{1}^{(n)}\right)^{\prime} \\
& =U_{i}-\frac{\sum_{k=1}^{n} Z_{k}^{2} \sum_{j=1}^{n} U_{j}-\sum_{k=1}^{n} Z_{k} \sum_{j=1}^{n} U_{j} Z_{j}}{n \sum_{j=1}^{n} Z_{j}^{2}-\left(\sum_{j=1}^{n} Z_{j}\right)^{2}}
\end{aligned}
$$




$$
-Z_{i} \frac{n \sum_{k=1}^{n} U_{k} Z_{k}-\sum_{k=1}^{n} U_{k} \sum_{j=1}^{n} Z_{j}}{n \sum_{j=1}^{n} Z_{j}^{2}-\left(\sum_{j=1}^{n} Z_{j}\right)^{2}}, i=1, \ldots, n .
$$

Therefore, for $0 \leq \xi \leq 1$,

$$
\begin{aligned}
\sum_{i=1}^{[n \xi]} U_{i}^{(n)}= & \sum_{i=1}^{[n \xi]} U_{i}-[n \xi] \frac{\sum_{k=1}^{n} Z_{k}^{2} \sum_{j=1}^{n} U_{j}-\sum_{k=1}^{n} Z_{k} \sum_{j=1}^{n} U_{j} Z_{j}}{n \sum_{j=1}^{n} Z_{j}^{2}-\left(\sum_{j=1}^{n} Z_{j}\right)^{2}} \\
& -\sum_{i=1}^{[n \xi]} Z_{i} \frac{n \sum_{k=1}^{n} U_{k} Z_{k}-\sum_{k=1}^{n} U_{k} \sum_{j=1}^{n} Z_{j}}{n \sum_{j=1}^{n} Z_{j}^{2}-\left(\sum_{j=1}^{n} Z_{j}\right)^{2}} \\
= & \left(\sum_{i=1}^{[n \xi]} U_{i}-\frac{[n \xi]}{n} \sum_{i=1}^{n} U_{i}\right) \\
& +\frac{[n \xi] \sum_{k=1}^{n} Z_{k} \sum_{j=1}^{n} U_{j} Z_{j}-n \sum_{i=1}^{[n \xi]} Z_{i} \sum_{k=1}^{n} U_{k} Z_{k}}{n \sum_{j=1}^{n} Z_{j}^{2}-\left(\sum_{j=1}^{n} Z_{j}\right)^{2}} \\
& +\frac{\sum_{i=1}^{[n \xi]} Z_{i} \sum_{j=1}^{n} Z_{j} \sum_{k=1}^{n} U_{k}-\frac{[n \xi]}{n} \sum_{k=1}^{n} U_{k}\left(\sum_{j=1}^{n} Z_{j}\right)^{2}}{n \sum_{j=1}^{n} Z_{j}^{2}-\left(\sum_{j=1}^{n} Z_{j}\right)^{2}} \\
=: & \left(\sum_{i=1}^{[n \xi]} U_{i}-\frac{[n \xi]}{n} \sum_{i=1}^{n} U_{i}\right)+I_{1}^{(n)}(\xi)+I_{2}^{(n)}(\xi) .
\end{aligned}
$$

We rewrite the process defined in $(23)$ as

$$
X_{n}(\xi)=\frac{a_{n}^{-1} \sum_{i=1}^{[n \xi]} U_{i}^{(n)}}{\left(a_{n}^{-2} \sum_{i=1}^{n} U_{i}^{(n) 2}\right)^{1 / 2}}, \quad 0 \leq \xi \leq 1, n \geq 1
$$

where $\left(a_{n}, n \geq 1\right)$ are given by (6). By (30), the numerator of (31) has the representation

$$
\begin{aligned}
\frac{1}{a_{n}} \sum_{i=1}^{[n \xi]} U_{i}^{(n)}= & \left(\frac{1}{a_{n}} \sum_{i=1}^{[n \xi]} U_{i}-\frac{1}{a_{n}} \xi \sum_{i=1}^{n} U_{i}\right) \\
& +\frac{1}{a_{n}}\left(\xi-\frac{[n \xi]}{n}\right) \sum_{i=1}^{n} U_{i}+\frac{1}{a_{n}} I_{1}^{(n)}(\xi)+\frac{1}{a_{n}} I_{2}^{(n)}(\xi) \\
= & \left(I_{\xi}\left(\mathbf{X}_{n}^{*}\right)-\xi I_{1}\left(\mathbf{X}_{n}^{*}\right)\right)+\frac{1}{a_{n}}\left(\xi-\frac{[n \xi]}{n}\right) \sum_{i=1}^{n} U_{i} \\
& +\frac{1}{a_{n}} I_{1}^{(n)}(\xi)+\frac{1}{a_{n}} I_{2}^{(n)}(\xi),
\end{aligned}
$$

where $\left(\mathbf{X}_{n}^{*}\right)$ is given by the right-hand side of (12), and 


$$
I_{\xi}(\mathbf{X}):=\int_{t \leq \xi} j d \mathbf{X}, \quad 0 \leq \xi \leq 1
$$

for all measures $\mathbf{X}$ on $[0, \infty) \times E$ for which the integral is well defined. Observe that for every fixed $\xi \in[0,1], \xi-\frac{[n \xi]}{n} \rightarrow_{n \rightarrow \infty} 0$. Furthermore, the sequence $\left(\frac{1}{a_{n}} \sum_{i=1}^{n} U_{i}\right)_{n \geq 1}$ is tight, ${ }^{10}$ which follows from Lemma 1 below. Therefore,

$$
\frac{1}{a_{n}}\left(\xi-\frac{[n \xi]}{n}\right) \sum_{i=1}^{n} U_{i} \stackrel{p}{\rightarrow}_{n \rightarrow \infty} 0
$$

where $\stackrel{p}{\rightarrow}$ stands for convergence in probability.

Considering the denominator in (31), we have

$$
\begin{aligned}
\sum_{i=1}^{n}\left(U_{i}^{(n)}\right)^{2} & =\sum_{i=1}^{n} U_{i}^{2}+\frac{n\left(\sum_{k=1}^{n} Z_{k}^{2} \sum_{j=1}^{n} U_{j}-\sum_{k=1}^{n} Z_{k} \sum_{j=1}^{n} U_{j} Z_{j}\right)^{2}}{n\left(\sum_{j=1}^{n} Z_{j}^{2}-\left(\sum_{j=1}^{n} Z_{j}\right)^{2}\right)^{2}} \\
& +\frac{\sum_{i=1}^{n} Z_{i}^{2}\left(n \sum_{k=1}^{n} U_{k} Z_{k}-\sum_{k=1}^{n} U_{k} \sum_{j=1}^{n} Z_{j}\right)^{2}}{\left(n \sum_{j=1}^{n} Z_{j}^{2}-\left(\sum_{j=1}^{n} Z_{j}\right)^{2}\right)^{2}} \\
& -\frac{2 \sum_{i=1}^{n} U_{i}\left(\sum_{k=1}^{n} Z_{k}^{2} \sum_{j=1}^{n} U_{j}-\sum_{k=1}^{n} Z_{k} \sum_{j=1}^{n} U_{j} Z_{j}\right)}{n \sum_{j=1}^{n} Z_{j}^{2}-\left(\sum_{j=1}^{n} Z_{j}\right)^{2}} \\
& -\frac{2 \sum_{i=1}^{n} U_{i} Z_{i}\left(n \sum_{k=1}^{n} U_{k} Z_{k}-\sum_{k=1}^{n} U_{k} \sum_{j=1}^{n} Z_{j}\right)}{n \sum_{j=1}^{n} Z_{j}^{2}-\left(\sum_{j=1}^{n} Z_{j}\right)^{2}} \\
& +\frac{2 \sum_{i=1}^{n} Z_{i}\left(\sum_{k=1}^{n} Z_{k}^{2} \sum_{j=1}^{n} U_{j}-\sum_{k=1}^{n} Z_{k} \sum_{j=1}^{n} U_{j} Z_{j}\right)}{n \sum_{j=1}^{n} Z_{j}^{2}-\left(\sum_{j=1}^{n} Z_{j}\right)^{2}} \\
& \times \frac{n \sum_{u=1}^{n} U_{k} Z_{k}-\sum_{k=1}^{n} U_{k} \sum_{j=1}^{n} Z_{j}}{n \sum_{j=1}^{n} Z_{j}^{2}-\left(\sum_{j=1}^{n} Z_{j}\right)^{2}} \\
= & \sum_{i=1}^{n} U_{i}^{2}+\sum_{j=1}^{5} R_{j}(n) .
\end{aligned}
$$

To continue the analysis of $\sum_{i=1}^{n}\left(U_{i}^{(n)}\right)^{2}$ we need the following lemma, whose the proof is given in the Appendix.

Lemma 1. Let $\left(V_{i}\right)_{i \geq 1}$ be i.i.d. r.v.'s such that for some $0<\alpha<2$ and $c>0$

$$
P\left(\left|V_{i}\right|>\lambda\right) \leq c \lambda^{-\alpha}, \quad \lambda>0,
$$

\footnotetext{
${ }^{10} \mathrm{~A}$ sequence of r.v.'s $\left(\eta_{i}, i \geq 1\right)$, is tight, if for every $\epsilon>0$ there exists a constant $K_{\epsilon}>0$, such that $\sup _{i \geq 1} P\left(\left|\eta_{i}\right|>K_{\epsilon}\right)<\epsilon$ (see, for example, Billingsley (1968)).
} 
for all $i \geq 1$. Let $\left(\xi_{i}\right)_{i \geq 1}$ be a sequence of real numbers such that

$$
\overline{\lim }_{n \rightarrow \infty} \frac{1}{n} \sum_{j=1}^{n}\left|\xi_{j}\right|^{2}<\infty
$$

(i) If $0<\alpha<1$, then

$$
\text { the sequence }\left(n^{-1 / \alpha} \sum_{j=1}^{n} V_{j} \xi_{j}\right), n \geq 1 \text {, is tight. }
$$

(ii) If $\alpha=1$, and

$$
\int_{-n}^{n} x d F_{V_{n}}(x) \rightarrow_{n \rightarrow \infty} 0
$$

then (38) holds.

(iii) If $1<\alpha<2$ and $E\left(V_{j}\right)=0$, then (38) holds.

For $0 \leq \xi \leq 1$ let

$$
T_{n}(\xi):=\frac{a_{n}^{-1} \sum_{i=1}^{[n \xi \xi} U_{i}^{(n)}}{\left(a_{n}^{-2} \sum_{i=1}^{n} U_{i}^{2}\right)^{1 / 2}} .
$$

Then, by the decomposition (32),

$$
\begin{aligned}
T_{n}(\xi)= & \frac{I_{\xi}\left(\mathbf{X}_{n}^{*}\right)-\xi I_{1}\left(\mathbf{X}_{n}^{*}\right)}{\left(a_{n}^{-2} \sum_{i=1}^{n} U_{i}^{2}\right)^{1 / 2}}+\frac{\frac{1}{a_{n}}\left(\xi-\frac{[n \xi]}{n}\right) \sum_{i=1}^{n} U_{i}}{\left(a_{n}^{-2} \sum_{i=1}^{n} U_{i}^{2}\right)^{1 / 2}} \\
& +\frac{\frac{1}{a_{n}} I_{1}^{(n)}(\xi)}{\left(a_{n}^{-2} \sum_{i=1}^{n} U_{i}^{2}\right)^{1 / 2}}+\frac{\frac{1}{a_{n}} I_{2}^{(n)}(\xi)}{\left(a_{n}^{-2} \sum_{i=1}^{n} U_{i}^{2}\right)^{1 / 2}} \\
=: & H_{n}(\xi)+R_{1}^{(n)}(\xi)+R_{2}^{(n)}(\xi)+R_{3}^{(n)}(\xi) .
\end{aligned}
$$

Lemma 2. As $n \rightarrow \infty$,

$$
R_{i}^{(n)}(\xi) \stackrel{p}{\rightarrow}_{n \rightarrow \infty} 0
$$

for all $i=1,2,3$ and for all $0 \leq \xi \leq 1$.

The proof is given in the Appendix. 
For any $0<a<b<\infty$, let $I_{a, b}^{(2)}(\mathbf{X})$ be defined on $M_{p}([0, \infty] \times([-\infty, \infty] \backslash\{0\}))$ as

$$
I_{a, b}^{(2)}(\mathbf{X}):=\int_{t \leq 1} j^{2} \mathbf{1}_{\{a \leq|j| \leq b\}} d \mathbf{X}
$$

and let $I^{(2)}\left(\mathbf{X}=\lim _{a \rightarrow 0, b \rightarrow \infty} I_{a, b}^{(2)}(\mathbf{X})\right.$. Define, similarly, for $0 \leq \xi \leq 1$,

$$
I_{a, b ; \xi}^{(1)}(\mathbf{X}):=\int_{t \leq \xi} j \mathbf{1}_{\{a \leq|j| \leq b\}} d \mathbf{X} .
$$

It is well known (Resnick, 1987) that $I_{a, b ; \xi}^{(1)}$ is a map $M_{p}([0, \infty] \times([-\infty, \infty] \backslash\{0\})) \rightarrow \mathbf{R}$ that is almost surely continuous with respect to the law of $\mathbf{X}^{*}$ (see (12)). Fix now a small $\gamma>0$, and let

$$
H_{a, b ; \xi ; \gamma}(\mathbf{X})=\frac{I_{a, b ; \xi}^{(1)}(\mathbf{X})-\xi I_{a, b ; 1}^{(1)}(\mathbf{X})}{\left(I_{a, b}^{(2)}(\mathbf{X})\right)^{1 / 2}+\gamma} .
$$

Then, $H_{a, b ; \xi ; \gamma}$ is a functional $M_{p}([0, \infty] \times([-\infty, \infty] \backslash\{0\}))$ which is almost surely continuous with respect to the law of $\mathbf{X}^{*}$. For arbitrary $0 \leq \xi_{1}<\ldots<\xi_{k} \leq 1$, the functional

$$
\begin{aligned}
& H_{a, b ; \gamma}^{(k)}\left(\xi_{1}, \ldots, \xi_{k} ; \mathbf{X}\right) \\
& \quad:=\left(H_{a, b ; \xi ; \gamma}\left(\xi_{1}\right), \ldots, H_{a, b ; \xi ; \gamma}\left(\xi_{k}\right)\right): M_{p}([0, \infty] \times([-\infty, \infty] \backslash\{0\})) \rightarrow \mathbf{R}^{k}
\end{aligned}
$$

is almost surely continuous. By the Continuous Mapping Theorem, we have

$$
\begin{aligned}
& \left(H_{n}^{a, b ; \gamma}\left(\xi_{1}\right), \ldots, H_{n}^{a, b ; \gamma}\left(\xi_{k}\right)\right) \\
& \quad \stackrel{щ}{\Rightarrow}_{n \rightarrow \infty}\left(\frac{I_{a, b ; \xi_{1}}^{(1)}\left(\mathbf{X}^{*}\right)-\xi_{1} I_{a, b ; 1}^{(1)}\left(\mathbf{X}^{*}\right)}{\left(I_{a, b}^{(2)}\left(\mathbf{X}^{*}\right)\right)^{1 / 2}+\gamma}, \ldots, \frac{I_{a, b ; \xi_{k}}^{(1)}\left(\mathbf{X}^{*}\right)-\xi_{k} I_{a, b ; 1}^{(1)}\left(\mathbf{X}^{*}\right)}{\left(I_{a, b}^{(2)}\left(\mathbf{X}^{*}\right)\right)^{1 / 2}+\gamma}\right)
\end{aligned}
$$

in $\mathbf{R}^{k}$, where for $0 \leq \xi \leq 1$,

$$
H_{n}^{a, b ; \gamma}(\xi):=H_{a, b ; \xi ; \gamma}\left(\mathbf{X}_{n}^{*}\right)
$$

and $\mathbf{X}_{n}^{*}$ is defined in (12). 
It turns out that $a, b$ and $\gamma$ can be set to $a=0, b=\infty$ and $\gamma=0$ (and, thus, we can replace $H_{n}^{a, b ; \gamma}(\xi)$ by $H_{n}(\xi)$ defined in $\left.(40)\right)$. This is shown in the following lemma.

Lemma 3. As $n \rightarrow \infty$,

$$
\left(H_{n}\left(\xi_{1}\right), \ldots, H_{n}\left(\xi_{k}\right)\right) \stackrel{m}{\Rightarrow}_{n \rightarrow \infty}\left(\frac{I_{\xi_{1}}\left(\mathbf{X}^{*}\right)}{\left(I^{(2)}\left(\mathbf{X}^{*}\right)\right)^{1 / 2}}, \ldots, \frac{I_{\xi_{k}}\left(\mathbf{X}^{*}\right)}{\left(I^{(2)}\left(\mathbf{X}^{*}\right)\right)^{1 / 2}}\right)
$$

in $\mathbf{R}^{k}$, where

$$
I_{\xi}\left(\mathbf{X}^{*}\right):=\sum_{j=1}^{\infty} \delta_{j} \Gamma_{j}^{-1 / \alpha}\left(\mathbf{1}_{\left\{U_{j}^{(0)} \leq \xi\right\}}-\xi\right), 0 \leq \xi \leq 1
$$

and the sequences $\left(\delta_{j}\right)_{j \geq 1},\left(\Gamma_{j}\right)_{j \geq 1}$ and $\left(U_{j}^{(0)}\right)_{j \geq 1}$ are defined in (24).

The proof is given in the Appendix.

From (40), Lemma 2 and Lemma 3 it follows that

$$
\left(T_{n}\left(\xi_{1}\right), \ldots, T_{n}\left(\xi_{k}\right)\right) \stackrel{щ}{\Rightarrow}_{n \rightarrow \infty}\left(\frac{I_{\xi_{1}}\left(\mathbf{X}^{*}\right)}{I^{(2)}\left(\mathbf{X}^{*}\right)^{1 / 2}}, \ldots, \frac{I_{\xi_{k}}\left(\mathbf{X}^{*}\right)}{I^{(2)}\left(\mathbf{X}^{*}\right)^{1 / 2}}\right)
$$

Since the coordinates of the vector in the right-hand side of (50) are almost surely non-zero, we also have

$$
\left(\frac{1}{T_{n}\left(\xi_{1}\right)}, \ldots, \frac{1}{T_{n}\left(\xi_{k}\right)}\right) \stackrel{щ}{\Rightarrow}_{n \rightarrow \infty}\left(\frac{I^{(2)}\left(\mathbf{X}^{*}\right)^{1 / 2}}{I_{\xi_{1}}\left(\mathbf{X}^{*}\right)}, \ldots, \frac{I^{(2)}\left(\mathbf{X}^{*}\right)^{1 / 2}}{I_{\xi_{k}}\left(\mathbf{X}^{*}\right)}\right)
$$

Next, we replace in the above limiting relation $T_{n}\left(\xi_{i}\right)$ by $X_{n}\left(\xi_{i}\right)$ as given in (31). Arguments similar to those used in the Appendix to prove Lemma 2 imply that

$$
a_{n}^{-2} R_{j}(n) \stackrel{p}{\rightarrow}_{n \rightarrow \infty} 0, \text { for all } j=1, \ldots, 5,
$$

with $R_{j}(n)$ as in $(35)$. Therefore,

$$
\begin{aligned}
\|\left(\frac{1}{X_{n}\left(\xi_{1}\right)}, \ldots,\right. & \left.\frac{1}{X_{n}\left(\xi_{k}\right)}\right)\left(\frac{1}{T_{n}\left(\xi_{1}\right)}, \ldots, \frac{1}{T_{n}\left(\xi_{k}\right)}\right) \|^{2} \\
& \leq \sum_{j=1}^{5}\left|a_{n}^{-2} R_{j}(n)\right| \sum_{m=1}^{k} \frac{1}{\left|a_{n}^{-1} \sum_{i=1}^{\left[n \xi_{m}\right]} U_{i}^{(n)}\right|^{2}} \stackrel{p}{\rightarrow}_{n \rightarrow \infty} 0,
\end{aligned}
$$


because, for each $m=1, \ldots, k,\left|\sum_{i=1}^{\left[n \xi_{m}\right]} U_{i}^{(n)} / a_{n}\right|$ converges weakly to an almost surely positive limit.

We conclude from (51) and (53) that

$$
\left(\frac{1}{X_{n}\left(\xi_{1}\right)}, \ldots, \frac{1}{X_{n}\left(\xi_{k}\right)}\right) \stackrel{щ}{\rightrightarrows}_{n \rightarrow \infty}\left(\frac{\left(I^{(2)}\left(\mathbf{X}^{*}\right)\right)^{1 / 2}}{I_{\xi_{1}}\left(\mathbf{X}^{*}\right)}, \ldots, \frac{I^{(2)}\left(\mathbf{X}^{*}\right)^{1 / 2}}{I_{\xi_{k}}\left(\mathbf{X}^{*}\right)}\right)
$$

which implies, as above, that

$$
\left(X_{n}\left(\xi_{1}\right), \ldots, X_{n}\left(\xi_{k}\right)\right) \stackrel{щ}{\Rightarrow}_{n \rightarrow \infty}\left(\frac{I_{\xi_{1}}\left(\mathbf{X}^{*}\right)}{I^{(2)}\left(\mathbf{X}^{*}\right)^{1 / 2}}, \ldots, \frac{I_{\xi_{k}}\left(\mathbf{X}^{*}\right)}{I^{(2)}\left(\mathbf{X}^{*}\right)^{1 / 2}}\right)
$$

We have now established that

$$
\left(X_{n}(\xi)\right)_{0 \leq \xi \leq 1} \stackrel{w}{\Rightarrow}_{n \rightarrow \infty}\left(\frac{I_{\xi}\left(\mathbf{X}^{*}\right)}{I^{(2)}\left(\mathbf{X}^{*}\right)^{1 / 2}}\right)_{0 \leq \xi \leq 1}
$$

in the sense of convergence of the finite-dimensional distributions. Recalling representation (14) of the the points of $\mathbf{X}^{*}$, we immediately see that

$$
\left(\frac{I_{\xi}\left(\mathbf{X}^{*}\right)}{I^{(2)}\left(\mathbf{X}^{*}\right)^{1 / 2}}\right)_{0 \leq \xi \leq 1} \stackrel{d}{=}\left(X_{\infty}(\xi)\right)_{0 \leq \xi \leq 1}
$$

with $X_{\infty}(\xi)$ defined in (24). Therefore, it remains to prove that (56) also holds in the sense of weak convergence in the $J_{1}$-topology in $D([0,1])$. Since we have already proved the convergence of finite-dimensional distributions, it remains only to prove tightness. This follows from Lemma 4, which is proved in the Appendix.

Lemma 4. The sequence $\left(\left\{X_{n}(\xi), 0 \leq \xi \leq 1\right\}, n \geq 1\right)$, is tight in $D([0,1])$.

Lemma 4 completes the proof of Theorem 1.

\section{Simulation Results}

It is common practice to approximate the finite-sample distribution of a test statistic by its limiting distribution. The functional limit theorem proved in the previous section allows us to construct tests for the constancy of regression coefficient $\beta$ by comparing 
the distribution of the estimated residuals, $U_{i}^{(n)}$, with that implied by the constantcoefficient assumption. The only condition our test statistic has to satisfy is that it is a functional of $\left(X_{n}(\xi), 0 \leq \xi \leq 1\right)$ which is continuous in the Skorohod topology on $D[0,1]$, at least with probability 1 with respect to the law of the limiting process $\left(X_{\infty}(\xi), 0 \leq \xi \leq 1\right)$. One then derives the distribution of the same test-statistic functional evaluated on the limiting process. In the presence of heavy-tailed disturbances the limiting process $\left(X_{\infty}(\xi), 0 \leq \xi \leq 1\right)$ is not a standard one. Because of its complicated probabilistic structure, one has to resort to simulations to tabulate distribution or density values. In this section we present simulation results for the marginal distributions of the limiting process of interest. It turns out that already for a sample size of $n=100$ the finite-sample distributions are reasonably well approximated by the limiting distributions.

\subsection{Limiting and Finite-sample Marginal Distributions}

We simulated 10,000 replications of $X_{\infty}(\xi)$ for $\xi=.01, .02, \ldots, .99$, truncating the infinite sums in (24) at 1000. The inclusion of additional summands had no noticeable impact on the approximations. For the corresponding finite-sample distributions of $X_{n}(\xi)$ we also simulated 10,000 replications with $n=100$. For $\alpha=1.1,1.5,1.9$, Figures 1-3 show the estimated densities of the finite-sample distributions (top graphs) and the approximate limit distributions (bottom graphs) as a function of $\xi .^{11}$

\subsection{Critical Values for Tests Based on Marginal Distributions}

To derive critical values we simulated the finite-sample distribution with $U_{i}$ being drawn from symmetric $\alpha$-stable distributions with $\alpha \in\{1.0,1.1, \ldots, 1.9,2.0\} .{ }^{12}$ Given the closeness of finite-sample and limiting distributions, we simulated $\mathbf{X}_{n}(\xi)$ ( see (23)) with sample size $n=100$, in order to keep the computational burden manageable. ${ }^{13}$

\footnotetext{
${ }^{11}$ The distributions become highly peaked as $\xi$ approaches 0 and 1 . This is especially the case for small $\alpha$ 's. Therefore, Figure 1 displays only the results for $\xi \in[.1, .9]$.

${ }^{12}$ We have confined our simulation studies to this $\alpha$ range, because it covers the $\alpha$ estimates reported in empirical work.

${ }^{13}$ As simulations show, increasing the sample size has no noticeable impact on the simulated critical values.
} 
Because $\mathbf{X}_{n}(\xi) \stackrel{d}{=} \mathbf{X}_{n}(1-\xi), \xi \in[0,1]$, we can restrict ourselves to $\xi \in[0,1 / 2]$. Specifically, we considered values $\xi \in\{0,0.01, \ldots, 0.49,0.5\}$. For each of the resulting $561(\alpha, \xi)$-combinations we simulated 20,000 replications of $\mathbf{X}_{n}(\xi)$.

Instead of tabulating the critical values for selected values of $\alpha$ and $\xi$, we use response-surface techniques to compactly summarize the simulation results. ${ }^{14}$ Another advantage of this approach is that it allows us to approximate critical values for intermediate $\alpha$ - and $\xi$-values. We consider the significance levels $1-\gamma$, with $\gamma=.01, .05, .10$, and fit to each of the three sets of $561(\alpha, \xi)$-combinations a function of the form

$$
c v_{\gamma}\left(\alpha_{*}, \xi_{*}\right) \approx \sum_{i=0}^{I_{\gamma}} \sum_{j=1}^{J_{\gamma}} c_{\gamma, i, j} \alpha_{*}^{i} \xi_{*}^{j}
$$

where

$$
\begin{aligned}
& \alpha_{*}=(\ln \alpha)^{1.15}, \\
& \xi_{*}=(\ln (1+\xi))^{1 / P_{\gamma}},
\end{aligned}
$$

with

$$
P_{\gamma}= \begin{cases}2, & \text { if } \gamma=.10 \\ 3, & \text { if } \gamma=.01, .05\end{cases}
$$

and

$$
\begin{aligned}
& I_{\gamma}= \begin{cases}1, & \text { if } \gamma=.05, .10 \\
2, & \text { if } \gamma=.01\end{cases} \\
& J_{\gamma}= \begin{cases}5, & \text { if } \gamma=.05, .10 \\
2, & \text { if } \gamma=.01\end{cases}
\end{aligned}
$$

The least-squares estimates of coefficients $c_{\gamma, i, j}$ are reported in Table 1 . Figure 4 compares simulated (left panel) and fitted (right panel) critical values and suggests a close fit. The good fit is also reflected by the adjusted $R^{2}$-values, which are .99972 , .99990 and .99993 for $\gamma=.01, .05, .10$, respectively. As is to be expected, the goodness of fit decreases somewhat as we move into the tail of the distribution, i.e., as $\gamma$ decreases.

\footnotetext{
${ }^{14}$ See Hendry (1984) and Myers, Khuri and Carter (1989) for details of the response-surface methodology.
} 


\section{Conclusions}

We have investigated the OLS-based CUSUM test for regressions with heavy-tailed disturbances. The resulting limiting distribution deviates substantially from that for the finite-variance case. Because the limiting process has a rather complicated structure, we resort to simulations to examine the limiting and prelimiting behavior as well as to obtain critical values for the test statistic. Using response-surface methods we derive simple polynomial approximations of critical values which involve only a dozen or less coefficients and, thus, can be easily implemented in applied work.

\section{Appendix}

Proof of Lemma 1. The case $0<\alpha<1$ : It follows from (36) that there are constants $a, b \in(0, \infty)$ such that

$$
\left|V_{j}\right| \stackrel{s t}{\leq} a S_{j}+b
$$

where $S_{j} \stackrel{d}{=} S_{\alpha}(1,1,0) .{ }^{15}$ Therefore,

$$
\begin{aligned}
n^{-1 / \alpha}\left|\sum_{j=1}^{n} V_{j} \xi_{j}\right| & \leq n^{-1 / \alpha} \sum_{j=1}^{n}\left|V_{j}\right|\left|\xi_{j}\right| \\
& \leq n^{-1 / \alpha} \sum_{j=1}^{n}\left(a S_{j}+b\right)\left|\xi_{j}\right| \\
& =a n^{-1 / \alpha} \sum_{j=1}^{n} S_{j}\left|\xi_{j}\right|+b n^{-1 / \alpha} \sum_{j=1}^{n}\left|\xi_{j}\right| .
\end{aligned}
$$

Now, by (37),

$$
n^{-1 / \alpha} \sum_{j=1}^{n}\left|\xi_{j}\right| \rightarrow_{n \rightarrow \infty} 0
$$

whereas

\footnotetext{
${ }^{15}$ We say that a r.v. $X$ is stochastically smaller than a r.v. $Y$ (denoted $X \stackrel{\text { st }}{\leq} Y$ ) if $P(X \geq x) \leq$ $P(Y \geq x)$ for all $x \in \mathbf{R}$.
} 


$$
n^{-1 / \alpha} \sum_{j=1}^{n} S_{j}\left|\xi_{j}\right| \stackrel{d}{=} S_{\alpha}\left(\left(\frac{1}{n} \sum_{j=1}^{n}\left|\xi_{j}\right|^{\alpha}\right)^{1 / \alpha}, 1,0\right)
$$

Since the scale parameter in the right hand side of (A.4) is bounded, we conclude that both terms on the right hand side of (A.2) are tight, and so the sequence $\left(n^{-1 / \alpha} \sum_{j=1}^{n} V_{j} \xi_{j}, n \geq 1\right)$ is itself tight.

The case $1<\alpha<2$ : Write

$$
X_{n}^{(1)}:=n^{-1 / \alpha} \sum_{i=1}^{n} \xi_{i} V_{i} \mathbf{1}\left(\left|V_{i}\right| \leq n^{1 / \alpha}\right), X_{n}^{(2)}:=n^{-1 / \alpha} \sum_{i=1}^{n} \xi_{i} V_{i} \mathbf{1}\left(\left|V_{i}\right|>n^{1 / \alpha}\right) .
$$

We have

$$
\begin{aligned}
E\left(X_{n}^{(1)}\right)^{2}= & n^{-2 / \alpha} E\left(V_{1}^{2} \mathbf{1}\left(\left|V_{1}\right| \leq n^{1 / \alpha}\right)\right) \sum_{i=1}^{n} \xi_{i}^{2} \\
& +n^{-2 / \alpha} E\left(V_{1} \mathbf{1}\left(\left|V_{1}\right| \leq n^{1 / \alpha}\right)\right)^{2} \sum_{1 \leq i \leq n, 1 \leq j \leq n, i \neq j} \xi_{i} \xi_{j} \\
\leq & n^{-2 / \alpha} E\left(V_{1}^{2} \mathbf{1}\left(\left|V_{1}\right| \leq n^{1 / \alpha}\right)\right) \sum_{i=1}^{n} \xi_{i}^{2} \\
& +n^{-2 / \alpha} \mathrm{E}\left(V_{1} \mathbf{1}\left(\left|V_{1}\right| \leq n^{1 / \alpha}\right)\right)^{2}\left(\sum_{i=1}^{n}\left|\xi_{i}\right|\right)^{2} .
\end{aligned}
$$

Now, by (36),

$$
\begin{aligned}
E\left(V_{1}^{2} \mathbf{1}\left(\left|V_{1}\right| \leq n^{1 / \alpha}\right)\right) & =\int_{0}^{\infty} P\left(V_{1}^{2} \mathbf{1}\left(\left|V_{1}\right| \leq n^{1 / \alpha}\right)>\lambda\right) d \lambda \\
& =\int_{0}^{n^{2 / \alpha}} P\left(\lambda^{1 / 2} \leq\left|V_{1}\right| \leq n^{1 / \alpha}\right) d \lambda \\
& \leq c \int_{0}^{n^{2 / \alpha}} \lambda^{-\alpha / 2} d \lambda \\
& =c n^{-1+2 / \alpha} .
\end{aligned}
$$

Here and in the sequel $c$ is some finite positive constant that may change from line to line. We conclude that there exists a constant $D_{1}<\infty$, such that for all $n \geq 1$,

$$
n^{-2 / \alpha} E\left(V_{1}^{2} \mathbf{1}\left(\left|V_{1}\right| \leq n^{1 / \alpha}\right)\right) \sum_{i=1}^{n} \xi_{i}^{2} \leq c \frac{1}{n} \sum_{i=1}^{n} \xi_{i}^{2} \leq D_{1}
$$


Furthermore, because $E V_{1}=0$,

$$
\begin{aligned}
\left|E\left(V_{1} \mathbf{1}\left(\left|V_{1}\right| \leq n^{1 / \alpha}\right)\right)\right| & =\left|E\left(V_{1} \mathbf{1}\left(\left|V_{1}\right|>n^{1 / \alpha}\right)\right)\right| \\
& \leq E\left(\left|V_{1}\right| \mathbf{1}\left(\left|V_{1}\right|>n^{1 / \alpha}\right)\right) \\
& =\int_{0}^{\infty} P\left(\left|V_{1}\right| \mathbf{1}_{\left\{\left|V_{1}\right|>n^{1 / \alpha}\right\}}>\lambda\right) d \lambda \\
& =n^{1 / \alpha} P\left(\left|V_{1}\right|>n^{1 / \alpha}\right)+\int_{n^{1 / \alpha}}^{\infty} P\left(\left|V_{1}\right|>\lambda\right) d \lambda \\
& \leq c n^{-1+1 / \alpha}+c \int_{n^{1 / \alpha}}^{\infty} \lambda^{-\alpha} d \lambda \\
& =c n^{-1+1 / \alpha} .
\end{aligned}
$$

Therefore, by (37),

$$
n^{-2 / \alpha}\left(E\left(V_{1} \mathbf{1}\left(\left|V_{1}\right| \leq n^{1 / \alpha}\right)\right)\right)^{2}\left(\sum_{i=1}^{n}\left|\xi_{i}\right|\right)^{2} \leq c\left(\frac{1}{n} \sum_{i=1}^{n}\left|\xi_{i}\right|\right)^{2} \leq D_{2}<\infty
$$

for some absolute constant $D_{2}$.

It follows now from (A.6), (A.7) and (A.9) that $\left(E\left(X_{n}^{(1)}\right)^{2}\right)_{n \geq 1}$ is a uniformly bounded sequence, and so

$$
\left(X_{n}^{(1)}\right)_{n \geq 1} \text { is tight. }
$$

Finally, by (A.8) we have for an absolute constant $D_{3}$,

$$
E\left|X_{n}^{(2)}\right| \leq n^{-1 / \alpha}\left(E\left(V_{1} \mathbf{1}\left(\left|V_{1}\right| \leq n^{1 / \alpha}\right)\right)\right) \sum_{i=1}^{n}\left|\xi_{i}\right| \leq c \frac{1}{n} \sum_{i=1}^{n}\left|\xi_{i}\right| \leq D_{3}<\infty
$$

This implies that

$$
\left(X_{n}^{(2)}, n \geq 1\right) \text { is tight; }
$$

and our statement follows in the case $1<\alpha<2$ from (A.5), (A.10) and (A.12).

The case $\alpha=1$ : We still use the decomposition (A.5). The same argument as in the case $1<\alpha<2$ shows that the sequence $\left(X_{n}^{(1)}, n \geq 1\right)$ is tight. Further, take any 
$0<\theta<1$, and choose a constant $b>0$, so large that

$$
P\left(\left|V_{i}\right|>b n \text { for some } i=1, \ldots, n\right) \leq \frac{\theta}{2}, \text { for all } n \geq 1
$$

Then, for every $M>0$

$$
P\left(\left|X_{n}^{(2)}\right| \geq M\right) \leq \frac{\theta}{2}+P\left(n^{-1} \sum_{i=1}^{n} \xi_{i} V_{i} \mathbf{1}\left(n<\left|V_{i}\right|<b n\right)>M\right)
$$

Now,

$$
\begin{aligned}
E\left|\frac{1}{n} \sum_{i=1}^{n} \xi_{i} V_{i} \mathbf{1}\left(n<\left|V_{i}\right|<b n\right)\right| & \leq \frac{1}{n} \sum_{i=1}^{n}\left|\xi_{i}\right| E\left(\left|V_{1}\right| \mathbf{1}\left(n<\left|V_{1}\right|<b n\right)\right) \\
& =\frac{1}{n} \sum_{i=1}^{n}\left|\xi_{i}\right| \int_{n}^{b n} x d F_{\left|V_{1}\right|}(x) \\
& \leq \frac{1}{n} \sum_{i=1}^{n}\left|\xi_{i}\right|\left(n P\left(\left|V_{1}\right|>n\right)\right. \\
& \left.+\int_{n}^{b n} P\left(\left|V_{1}\right|>y\right) d y\right) \\
& \leq \frac{1}{n} \sum_{i=1}^{n}\left|\xi_{i}\right|\left(c+c \int_{n}^{b n} \frac{d y}{y}\right) \\
& =c \frac{1}{n} \sum_{i=1}^{n}\left|\xi_{i}\right|(1+\log b) \leq c(b)<\infty
\end{aligned}
$$

Choosing $M \geq \frac{2 c(b)}{\theta}$, we have

$$
P\left(\left|X_{n}^{(2)}\right|>M\right) \leq \theta, \text { for all } n \geq 1
$$

Hence, the tightness property of $\left(X_{n}^{(2)}, n \geq 1\right)$ is established.

Proof of Lemma 2. In our notation, $a_{n}^{-2} \sum_{i=1}^{n} U_{i}^{2}=I^{(2)}\left(\mathbf{X}_{n}^{*}\right)$. It converges weakly to a positive r.v.. Therefore, $\left(I^{(2)}\left(\mathbf{X}_{n}^{*}\right)^{-1 / 2}, n \geq 1\right)$ is a tight sequence and, by (34),

$$
R_{1}^{(n)}(\xi) \stackrel{p}{\rightarrow}_{n \rightarrow \infty} 0, \text { for all } 0 \leq \xi \leq 1
$$

The remaining part of the lemma will follow once we prove that 


$$
\frac{1}{a_{n}} I_{i}^{(n)}(\xi) \stackrel{p}{\rightarrow}_{n \rightarrow \infty} 0 \text { for } i=1,2 \text { and all } 0 \leq \xi \leq 1
$$

We have by (17) and Lemma 1, that

$$
\left|\frac{[n \xi] \sum_{k=1}^{n} Z_{k} \sum_{j=1}^{n} U_{j} Z_{j}}{n^{2} n^{1 / \alpha}}\right| \leq \frac{1}{n}\left|\sum_{k=1}^{n} Z_{k}\right| n^{-1 / \alpha}\left|\sum_{j=1}^{n} U_{j} Z_{j}\right| \stackrel{p}{\rightarrow}_{n \rightarrow \infty} 0 .
$$

Similarly,

$$
\left|\frac{n \sum_{i=1}^{[n \xi]} Z_{i} \sum_{k=1}^{n} U_{k} Z_{k}}{n^{2} n^{1 / \alpha}}\right| \leq \frac{1}{[n \xi]}\left|\sum_{i=1}^{[n \xi]} Z_{i}\right| n^{-1 / \alpha}\left|\sum_{k=1}^{n} U_{k} Z_{k}\right| \rightarrow_{n \rightarrow \infty} 0 .
$$

Moreover, by (17) and (18),

$$
\frac{1}{n^{2}}\left|n \sum_{j=1}^{n} Z_{j}^{2}-\left(\sum_{j=1}^{n} Z_{j}\right)^{2}\right| \rightarrow_{n \rightarrow \infty} R>0
$$

Now, (A.14) with $i=1$ follows from (A.15)-(A.17). Furthermore,

$$
\left|\frac{\sum_{i=1}^{[n \xi]} Z_{i} \sum_{j=1}^{n} Z_{j} \sum_{k=1}^{n} U_{k}}{n^{2} n^{1 / \alpha}}\right| \leq \frac{1}{[n \xi]}\left|\sum_{i=1}^{[n \xi]} Z_{i}\right| \frac{1}{n}\left|\sum_{j=1}^{n} Z_{j}\right|\left|n^{-1 / \alpha} \sum_{k=1}^{n} U_{k}\right| \stackrel{p}{\rightarrow}_{n \rightarrow \infty} 0
$$

by (17) and because the sequence $\left(n^{-1 / \alpha} \sum_{k=1}^{n} U_{k}\right)_{n \geq 1}$ is tight.

Similarly,

$$
\left|\frac{\frac{[n \xi]}{n}\left(\sum_{j=1}^{n} Z_{j}\right)^{2} \sum_{k=1}^{n} U_{k}}{n^{2} n^{1 / \alpha}}\right| \leq\left(\frac{1}{n} \sum_{j=1}^{n} Z_{j}\right)^{2}\left|n^{-1 / \alpha} \sum_{k=1}^{n} U_{k}\right| \stackrel{p}{\rightarrow}_{n \rightarrow \infty} 0 .
$$

Therefore, we have (A.14) with $i=2$ by (A.17)-(A.19). 
Proof of Lemma 3. Observe that $I_{\xi}\left(\mathbf{X}^{*}\right)$ is well defined, and

$$
I_{a, a^{-1}, \xi}^{(1)}\left(\mathbf{X}^{*}\right)-\xi I_{a, a^{-1}, 1}^{(1)}\left(\mathbf{X}^{*}\right) \rightarrow_{a \rightarrow 0} I_{\xi}\left(\mathbf{X}^{*}\right) \quad \text { almost surely. }
$$

To prove the lemma we will use Theorem 4.2 of Billingsley (1968). The first step is to show that for any $\gamma>0$

$$
\begin{aligned}
& \left(\frac{\frac{1}{a_{n}} \sum_{i=1}^{\left[n \xi_{j}\right]} U_{i}-\xi_{j} \frac{1}{a_{n}} \sum_{i=1}^{n} U_{i}}{\left(a_{n}^{-2} \sum_{i=1}^{n} U_{i}^{2}\right)^{1 / 2}+\gamma}, j=1, \ldots, k\right) \\
& \stackrel{w}{\Rightarrow}_{n \rightarrow \infty}\left(\frac{I_{\xi_{j}}\left(\mathbf{X}^{*}\right)}{\left(I^{(2)}\left(\mathbf{X}^{*}\right)\right)^{1 / 2}+\gamma}, j=1, \ldots, k\right) .
\end{aligned}
$$

To this end it is enough to show that for every $0 \leq \xi \leq 1$,

$$
\lim _{a \rightarrow 0} \varlimsup_{n \rightarrow \infty} P\left(\left|\Delta_{n}(\xi)\right| \geq \varepsilon\right)=0 \text { for every } 0<\varepsilon<1 \text {, }
$$

where

$$
\begin{aligned}
\Delta_{n}(\xi)= & \frac{\frac{1}{a_{n}} \sum_{i=1}^{[n \xi]} U_{i} \mathbf{1}\left(a_{n} a<\left|U_{i}\right|<a_{n} a^{-1}\right)-\xi \frac{1}{a_{n}} \sum_{i=1}^{n} U_{i} \mathbf{1}\left(a_{n} a<\left|U_{i}\right|<a_{n} a^{-1}\right)}{\left(a_{n}^{-2} \sum_{i=1}^{n} U_{i}^{2} \mathbf{1}\left(a_{n} a<\left|U_{i}\right|<a_{n} a^{-1}\right)^{1 / 2}+\gamma\right)} \\
& -\frac{\frac{1}{a_{n}} \sum_{i=1}^{[n \xi]} U_{i}-\xi \frac{1}{a_{n}} \sum_{i=1}^{n} U_{i}}{\left(a_{n}^{-2} \sum_{i=1}^{n} U_{i}^{2}\right)^{1 / 2}+\gamma} .
\end{aligned}
$$

We have

$$
\begin{aligned}
P(|\Delta(\xi)| \geq \varepsilon) \leq P & \left(\mid \frac{\frac{1}{a_{n}} \sum_{i=1}^{[n \xi]} U_{i}\left(\mathbf{1}\left(\left|U_{i}\right|<a_{n} a\right)+\mathbf{1}\left(\left|U_{i}\right|>a_{n} a^{-1}\right)\right)}{\left(a_{n}^{-2} \sum_{i=1}^{n} U_{i}^{2} \mathbf{1}\left(a_{n} a<\left|U_{i}\right|<a_{n} a^{-1}\right)\right)^{1 / 2}+\gamma}\right. \\
& \left.-\frac{\xi \frac{1}{a_{n}} \sum_{i=1}^{n} U_{i}\left(\mathbf{1}\left(\left|U_{i}\right|<a_{n} a\right)+\mathbf{1}\left(\left|U_{i}\right| \geq a_{n} a^{-1}\right)\right)}{\left(a_{n}^{-2} \sum_{i=1}^{n} U_{i}^{2} \mathbf{1}\left(a_{n} a<\left|U_{i}\right|<a_{n} a^{-1}\right)\right)^{1 / 2}+\gamma} \geq \frac{\epsilon}{2} \mid\right)
\end{aligned}
$$




$$
\begin{aligned}
&+ P\left(\left|\frac{1}{a_{n}} \sum_{i=1}^{[n \xi]} U_{i}-\xi \frac{1}{a_{n}} \sum_{i=1}^{n} U_{i}\right| \mid \frac{1}{\left(a_{n}^{-2} \sum_{i=1}^{n} U_{i}^{2}\right)^{1 / 2}+\gamma}\right. \\
&\left.-\frac{1}{\left(a_{n}^{-2} \sum_{i=1}^{n} U_{i}^{2} \mathbf{1}\left(a_{n} a<\left|U_{i}\right|<a_{n} a^{-1}\right)\right)^{1 / 2}+\gamma} \mid \geq \frac{\epsilon}{2}\right) \\
&=: \quad q_{n}^{(1)}(a, \varepsilon)+q_{n}^{(2)}(a, \varepsilon) .
\end{aligned}
$$

Furthermore,

$$
\begin{aligned}
q_{n}^{(1)}(a, \varepsilon) & \leq P\left(\frac{1}{a_{n}}\left|\sum_{i=1}^{[n \xi]} U_{i} \mathbf{1}\left(\left|U_{i}\right|<a a_{n}\right)\right|>\frac{\varepsilon \gamma}{8}\right) \\
& +P\left(\frac{1}{a_{n}}\left|\sum_{i=1}^{[n \xi]} U_{i} \mathbf{1}\left(\left|U_{i}\right|>a^{-1} a_{n}\right)\right|>\frac{\varepsilon \gamma}{8}\right) \\
& +P\left(\frac{1}{a_{n}}\left|\sum_{i=1}^{n} U_{i} \mathbf{1}\left(\left|U_{i}\right|<a a_{n}\right)\right|>\frac{\varepsilon \gamma}{8}\right) \\
& \left.+P\left(\frac{1}{a_{n}}\left|\sum_{i=1}^{n} U_{i} \mathbf{1}_{(}\right| U_{i} \mid>a^{-1} a_{n}\right) \mid>\frac{\varepsilon \gamma}{8}\right) \\
& :=q_{n}^{(1,1)}\left(a, \frac{\varepsilon \gamma}{8}\right)+q_{n}^{(1,2)}\left(a, \frac{\varepsilon \gamma}{8}\right)+q_{n}^{(1,3)}\left(a, \frac{\varepsilon \gamma}{8}\right)+q_{n}^{(1,4)}\left(a, \frac{\varepsilon \gamma}{8}\right) .
\end{aligned}
$$

We claim that

$$
\lim _{a \rightarrow 0} \varlimsup_{n \rightarrow \infty} q_{n}^{(1, i)}(a, \varepsilon)=0, \text { for } i=1, \ldots, 4
$$

Clearly, (A.26) and (A.25) will imply that

$$
\lim _{a \rightarrow 0} \varlimsup_{n \rightarrow \infty} q_{n}^{(1)}(a, \varepsilon)=0
$$

We prove (A.26) only for $i=3,4$, as the other two cases are similar. The proof of (A.26) for $i=4$ follows from the following inequalities: for some constant $c>0$,

$$
\begin{aligned}
q_{n}^{(1,4)}(a, \epsilon) & \leq P\left(\text { at least one } U_{i}, i=1, \ldots, n, \text { satisfies }\left|U_{i}\right|>a^{-1} a_{n}\right) \\
& =1-\left(1-P\left(\left|U_{i}\right|>a^{-1} n^{1 / \alpha}\right)\right)^{n}
\end{aligned}
$$




$$
\leq 1-\left(1-c a^{\alpha} n^{-1}\right)^{n} \rightarrow_{n \rightarrow \infty} 1-e^{-c a^{\alpha}}
$$

We turn now to the proof of (A.26) with $i=3$. For the case $0<\alpha<1$ we use the inequalities

$$
\begin{aligned}
q_{n}^{(1,3)}(a, \epsilon) & \leq c \epsilon^{-1} n^{-1 / \alpha} n E\left(\left|U_{1}\right| \mathbf{1}\left(\left|U_{1}\right|<a a_{n}\right)\right) \\
& \leq c \epsilon^{-1} n^{-1 / \alpha+1} \int_{0}^{a n^{1 / \alpha}} P\left(\left|U_{1}\right|>y\right) d y \\
& \leq c \epsilon^{-1} n^{-1 / \alpha+1} \int_{0}^{a n^{1 / \alpha}} y^{-\alpha} d y \\
& =c \epsilon^{-1} a^{1-\alpha} .
\end{aligned}
$$

Thus, (A.26) holds for $i=3$.

Consider now the case $1<\alpha<2$. Repeating the computation used in the proof of Lemma 1, we have

$$
\begin{aligned}
E & \left(\frac{1}{n^{1 / \alpha}}\left|\sum_{i=1}^{n} U_{i} \mathbf{1}\left(\left|U_{i}\right|<a n^{1 / \alpha}\right)\right|\right)^{2} \\
& \leq n^{1-2 / \alpha}\left[E\left(U_{1}^{2} \mathbf{1}\left(\left|U_{i}\right|<a n^{1 / \alpha}\right)\right)+n\left(E\left(U_{1} \mathbf{1}\left(\left|U_{i}\right| \leq a n^{1 / \alpha}\right)\right)\right)^{2}\right] \\
& \leq c n^{1-2 / \alpha}\left[a^{2-\alpha} n^{-1+2 / \alpha}+n\left(a^{\alpha-1} n^{-1+1 / \alpha}\right)^{2}\right] \\
& \leq c\left(a^{2-\alpha}+a^{2 \alpha-2}\right)
\end{aligned}
$$

for some $0<c<\infty$. Therefore,

$$
\lim _{a \rightarrow 0} \varlimsup_{n \rightarrow \infty} E\left(\frac{1}{n^{1 / \alpha}}\left|\sum_{i=1}^{n} U_{i} \mathbf{1}\left(\left|U_{i}\right|<a n^{1 / \alpha}\right)\right|\right)^{2}=0
$$

implying (A.26) with $i=3$.

The case $\alpha=1$ remains. Here, we use assumption (A2) and repeat the computation in (A.30) above to obtain

$$
E\left(\frac{1}{n}\left|\sum_{i=1}^{n} U_{i} \mathbf{1}\left(\left|U_{i}\right| \leq a n\right)\right|\right)^{2} \leq c n^{-1}\left(a n+n\left(E\left(U_{1} \mathbf{1}\left(\left|U_{i}\right| \leq a n\right)\right)\right)^{2}\right) \rightarrow_{n \rightarrow \infty} c a
$$


Hence, (A.31) still holds and (A.26) has been proved for all cases.

If we show that

$$
\lim _{a \rightarrow 0} \varlimsup_{n \rightarrow \infty} q_{n}^{(2)}(\epsilon, i)=0
$$

then (A.24), (A.26) and (A.32) imply (A.22), and (A.21) will follow.

To this end, observe that, by Lemma 1, the sequence

$$
\left(\left|\frac{1}{a_{n}} \sum_{i=1}^{[n \xi]} U_{i}-\xi \frac{1}{a_{n}} \sum_{i=1}^{n} U_{i}\right|\right)_{n \geq 1}
$$

is tight. Therefore, (A.32) will follow once we prove that

$$
\lim _{a \rightarrow 0} \varlimsup_{n \rightarrow \infty} P\left(\left|\tilde{\Delta}_{n}\right|>\epsilon\right)=0
$$

where

$$
\tilde{\Delta}_{n}:=\frac{1}{\left(a_{n}^{-2} \sum_{i=1}^{n} U_{i}^{2}\right)^{1 / 2}+\gamma}-\frac{1}{\left(a_{n}^{-2} \sum_{i=1}^{n} U_{i}^{2} \mathbf{1}\left(a a_{n}<\left|U_{i}\right|<a^{-1} a_{n}\right)\right)^{1 / 2}+\gamma}
$$

However,

$$
\left|\tilde{\Delta}_{n}\right| \leq \gamma^{-2}\left(a_{n}^{-2} \sum_{i=1}^{n} U_{i}^{2}\left(\mathbf{1}\left(\left|U_{i}\right|<a a_{n}\right)+\mathbf{1}\left(\left|U_{i}\right|>a^{-1} a_{n}\right)\right)\right)
$$

and so (A.33) follows from the same arguments we used in proving (A.26) for $i=3,4$. Therefore, (A.21) follows.

We now turn to the proof of (48). Using once again Theorem 4.2 of Billingsley (1968) and (A.21), we conclude that it is enough to show that for every $0<\xi<1$, and for every $0<\varepsilon<1$, we have

$$
\begin{aligned}
\lim _{\gamma \rightarrow 0} \varlimsup_{n \rightarrow \infty} P\left(\mid \frac{\left(a_{n}^{-2} \sum_{i=1}^{n} U_{i}^{2}\right)^{1 / 2}+\gamma}{\frac{1}{a_{n}} \sum_{i=1}^{[n \xi]} U_{i}-\xi \frac{1}{a_{n}} \sum_{i=1}^{n} U_{i}}\right. \\
\left.-\frac{\left(a_{n}^{-2} \sum_{i=1}^{n} U_{i}^{2}\right)^{1 / 2}}{\frac{1}{a_{n}} \sum_{i=1}^{[n \xi]} U_{i}-\xi \frac{1}{a_{n}} \sum_{i=1}^{n} U_{i}} \mid>\varepsilon\right)=0 .
\end{aligned}
$$


However, (A.34) follows from the fact that $\left|a_{n}^{-1} \sum_{i=1}^{[n \xi]} U_{i}-\xi a_{n}^{-1} \sum_{i=1}^{n} U_{i}\right|$ converges weakly, as $n \rightarrow \infty$, to an almost surely positive limit. This completes the proof of Lemma 3.

Proof of Lemma 4. Observe that the denominator in (31) converges weakly to an almost surely positive limit. Therefore, it is enough to prove that the sequence of processes

$$
Y_{n}(\xi)=\left\{\frac{1}{a_{n}} \sum_{i=1}^{[n \xi]} U_{i}^{(n)}, 0 \leq \xi \leq 1\right\}
$$

is tight.

To this end we turn to (30). Taking (17) and (18), into account the tightness in (A.35) will follow once we prove the following statements:

$$
\begin{gathered}
\text { Sequence }\left\{a_{n}^{-1} \sum_{i=1}^{[n \xi]} U_{i}, 0 \leq \xi \leq 1\right\} \text { is tight; } \\
\text { Sequence }\left\{\left(a_{n}^{-1} \sum_{i=1}^{n} U_{i}\right) \frac{[n \xi]}{n}, 0 \leq \xi \leq 1\right\} \text { is tight; } \\
\text { Sequence }\left\{a_{n}^{-1} \frac{[n \xi] \sum_{k=1}^{n} Z_{k} \sum_{j=1}^{n} U_{j} Z_{j}-n \sum_{i=1}^{[n \xi]} Z_{i} \sum_{k=1}^{n} U_{k} Z_{k}}{n^{2}}, 0 \leq \xi \leq 1\right\}
\end{gathered}
$$

is tight;

$$
\text { Sequence }\left\{a_{n}^{-1} \frac{\sum_{i=1}^{[n \xi]} Z_{i} \sum_{j=1}^{n} Z_{j} \sum_{k=1}^{n} U_{k}-\frac{[n \xi]}{n}\left(\sum_{j=1}^{n} Z_{j}\right)^{2} \sum_{k=1}^{n} U_{k}}{n^{2}}, 0 \leq \xi \leq 1\right\}
$$

is tight.

Now, by the invariance principle, the sequence in (A.36) actually converges weakly in $D([0,1])$ and is, therefore, tight. Furthermore, $\left\{\frac{[n \xi]}{n}, 0 \leq \xi \leq 1\right\} \rightarrow\{\xi, 0 \leq \xi \leq 1\}$ in $D([0,1])$. Since $\left(a_{n}^{-1} \sum_{i=1}^{n} U_{i}, n \geq 1\right)$ is tight, (A.37) follows. An identical argument 
shows that the sequence

$$
\left\{a_{n}^{-1} \frac{[n \xi] \sum_{k=1}^{n} Z_{k} \sum_{j=1}^{n} U_{j} Z_{j}}{n^{2}}, 0 \leq \xi \leq 1\right\} \text { is tight. }
$$

Moreover, it follows from (17) that

$$
\sup _{0 \leq \xi \leq 1} \frac{1}{n}\left|\sum_{i=1}^{[n \xi]} Z_{i}\right| \rightarrow_{n \rightarrow \infty} 0
$$

Therefore, the sequence

$$
\left\{n \sum_{i=1}^{[n \xi]} Z_{i} \sum_{k=1}^{n} U_{k} Z_{k}, 0 \leq \xi \leq 1\right\}
$$

is tight. Now, (A.38) follows from (A.40) and (A.42). The proof of (A.39) uses the same arguments as the proof of (A.38). This proves Lemma 4.

\section{References}

[1] Billingsley, P. (1968), Convergence of Probability Measures, New York: Wiley \& Sons.

[2] Brown, R.L., J. Durbin and J.M Evans (1975), Techniques for testing the constancy of regression relationships over time, Journal of the Royal Statistical Society, Series B 37, 149-163.

[3] Fama, E. (1965), The behavior of stock market prices, Journal of Business 38, 34-105.

[4] Feller, W. (1971), An Introduction to Probability Theory and Its Applications Vol. 2 (2nd ed.), New York: Wiley.

[5] Hendry, D.A. (1984), Monte Carlo experimentation in econometrics, in: Z. Griliches and M.D. Intrilligator (ed.), Handbook of Econometrics, Vol. II, Ch. 16. Amsterdam.

[6] Kim, J.-R., S. Mittnik and S.T. Rachev (1996), The CUSUM test based on OLSresiduals when disturbances are heavy-tailed, Unpublished manuscript, Institute of Statistics and Econometrics, Christian Albrechts University at Kiel. 
[7] Lévy, P. (1937), Theorie de L'addition des Uariables Aleatories (2nd ed.), Paris: Gauthier-Uillars.

[8] Loretan, M. and P.C.B. Phillips (1994), Testing the covariance stationarity of heavy-tailed time series, Journal of Empirical Finance 1, 211-248.

[9] Mandelbrot, B. (1963), The variation of certain speculative prices, Journal of Business 26, 394-419.

[10] McCabe, B.P.M. and M.J. Harrison (1980), Testing the constancy of regression relationships over time using least squares residuals, Journal of the Royal Statistical Society, Series C 29, 142-148.

[11] McCulloch, J.H. (1996), Financial applications of stable distributions, in: Statistical Methods in Finance, Handbook of Statistics, Vol. 14, ed. G.S. Maddala and C.R. Rao, Elsevier Science.

[12] MacNeill, I.B. (1978), Properties of sequences of partial sums of polynomial regression residuals with applications to tests for change of regression at unknown times, The Annals of Statistics 6, 422-433.

[13] Mittnik, S. and S.T. Rachev (1993), Modeling asset returns with alternative stable distributions, Econometric Review 12, 261-330.

[14] Mittnik S. and S.T. Rachev (1998), Asset and Option Pricing with Alternative Stable Models, Series in Financial Economics and Quantitative Analysis, Wiley, forthcoming.

[15] Mittnik, S., S.T. Rachev, J.-R. Kim and (1998), Chi-square-type distributions for heavy-tailed variates, Econometric Theory 14, 339-354

[16] Mittnik, S., S.T. Rachev, and M. Paolella (1997), Stable Paretian modeling in finance: some empirical and theoretical aspects, in: A Practical Guide to Heavy Tails, ed. R.J. Adler, R.E. Feldman and M.S. Taqqu, Boston: Birkhäuser

[17] Myers, R.H., I. Khuri and W.-H. Carter, Jr. (1989), Response surface methodology: 1966-1988, Technometrics 31, 137-157.

[18] Ploberger, W. and W. Krämer (1992), The CUSUM test with OLS residuals, Econometrica 60, 271-285.

[19] Rachev, S.T., J.-R. Kim and S. Mittnik (1997), Econometric modeling in the presence of heavy-tailed innovations: a survey of some recent advances, Stochastic Models 13, 841-866.

[20] Resnick, S. (1987), Extreme Values, Regular Variation and Point Processes, New York: Springer-Verlag. 
[21] Samorodnitsky G. and M.S. Taqqu (1994), Stable Non-Gaussian Random Processes, New York: Chapman \& Hall.

[22] Zolotarev, V.M. (1986), One-dimensional Stable Distributions, Translations of Mathematical Monographs, American Mathematical Society, Vol. 65, Providence. 
Table 1. Coefficients of Response Surface Estimates $c_{\gamma, i, j}$ in Eqn. (58)

\begin{tabular}{|cc|ccccc|}
\hline & & \multicolumn{5}{|c|}{$j$} \\
& $i$ & 1 & 2 & 3 & 4 & 5 \\
\hline & 0 & -2.929 & 21.90 & -26.64 & -7.519 & 18.53 \\
.10 & 1 & 5.483 & -31.77 & 41.77 & 13.83 & -34.20 \\
& 2 & - & - & - & - & - \\
\hline & 0 & -8.926 & 87.85 & -248.5 & 295.0 & -127.6 \\
.05 & 1 & 15.22 & -137.9 & 404.7 & -487.4 & 210.3 \\
& 2 & - & - & - & - & - \\
\hline & 0 & 6.754 & -19.46 & 27.11 & -13.93 & - \\
.01 & 1 & -1.240 & 10.74 & -24.64 & 18.33 & - \\
& 2 & -11.71 & 33.28 & -19.85 & -5.538 & - \\
\hline
\end{tabular}


Figure 1. Simulated Finite-sample and Limit Distributions for $\alpha=1.1$

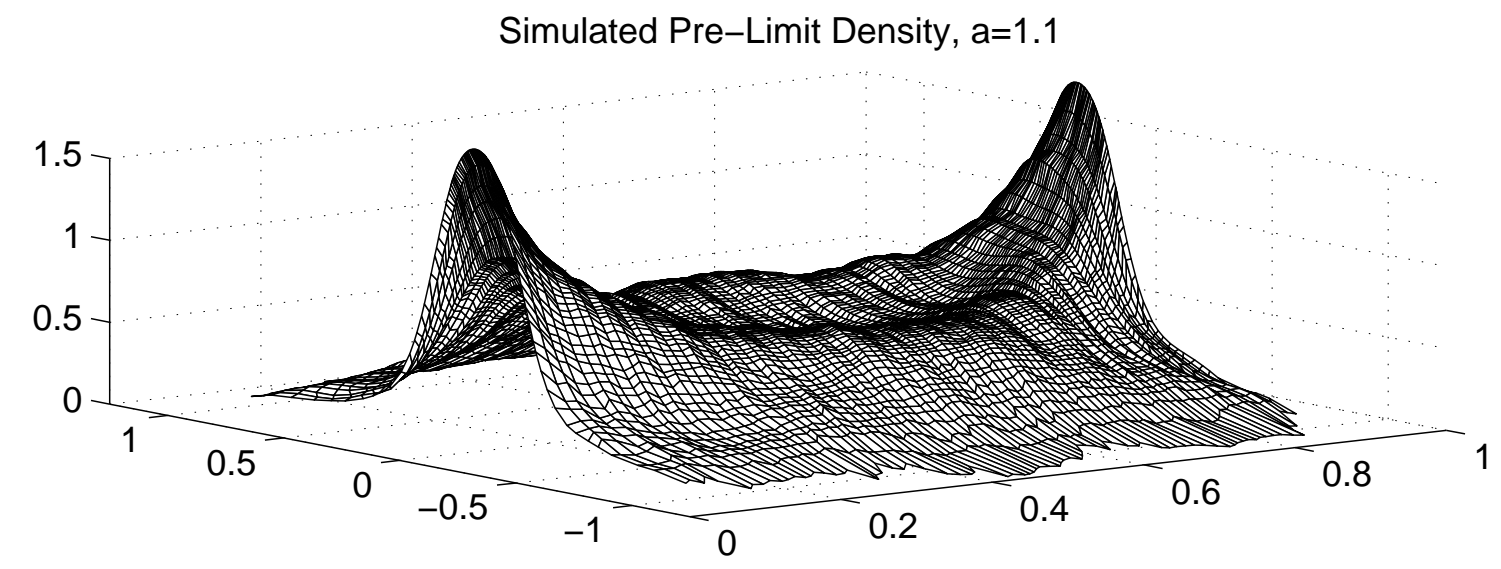

Simulated Limit Density, $a=1.1$

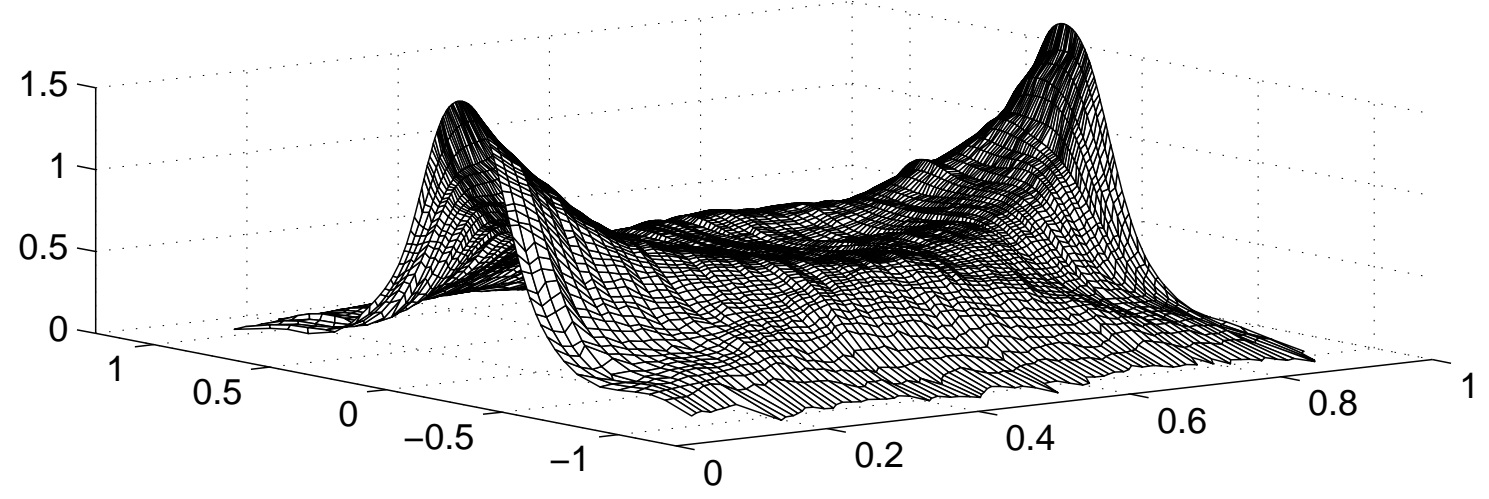


Figure 2. Simulated Finite-sample and Limit Distributions for $\alpha=1.5$

Simulated Pre-Limit Density, $\mathrm{a}=1.5$

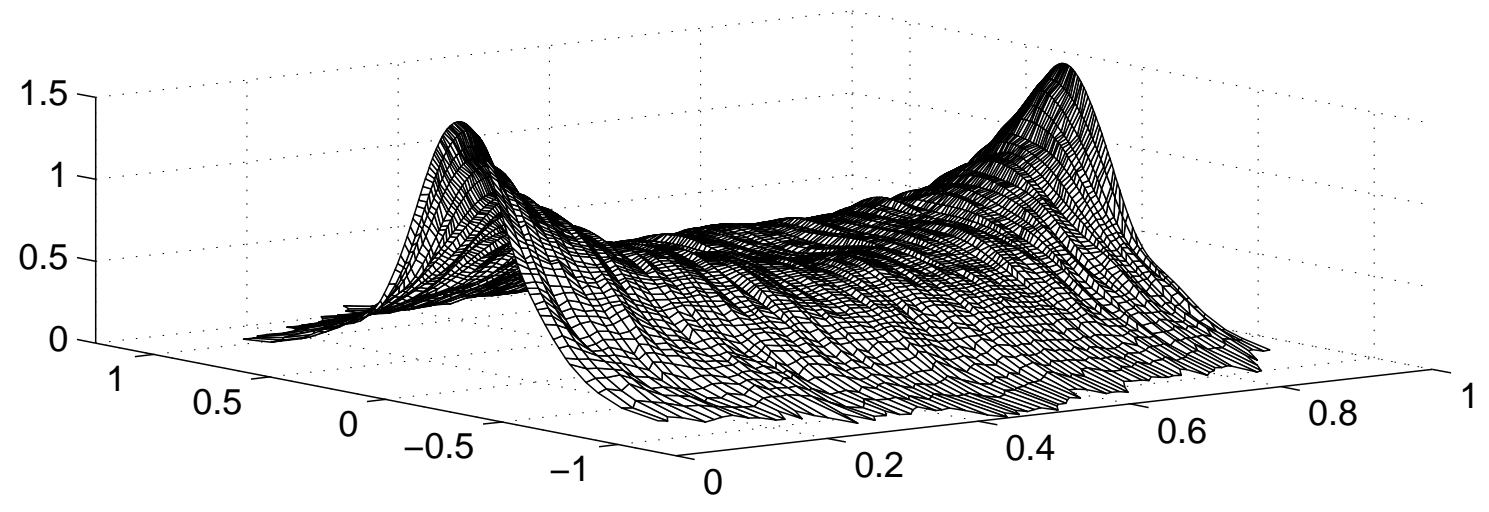

Simulated Limit Density, a=1.5

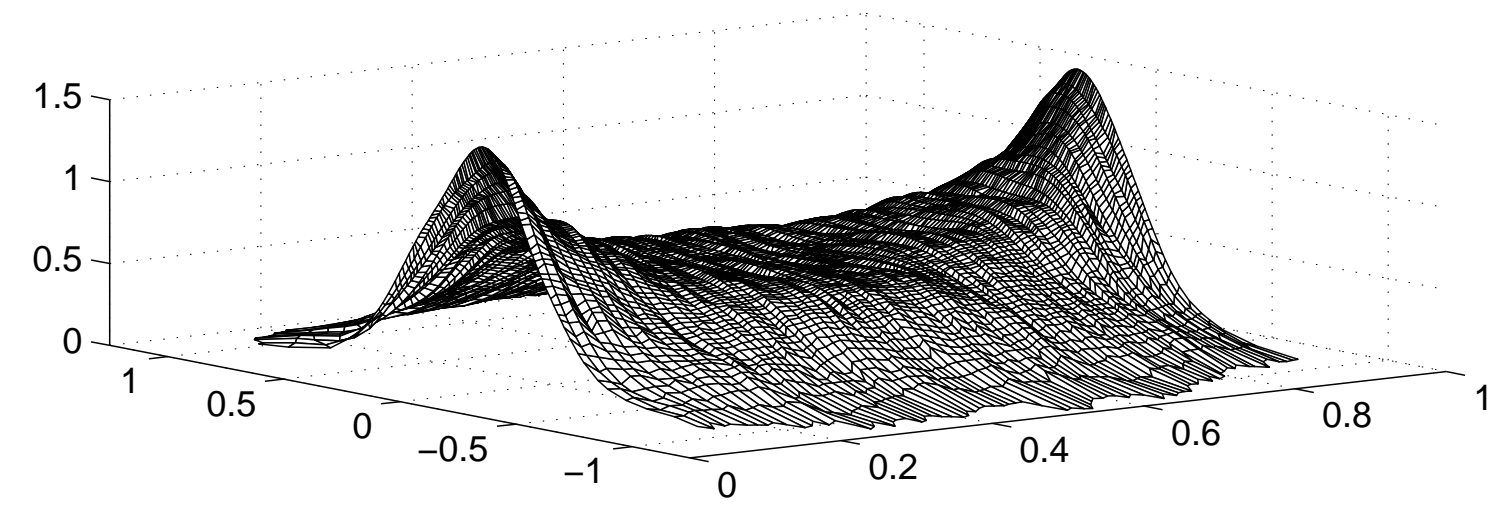


Figure 3. Simulated Finite-sample and Limit Distributions for $\alpha=1.9$

Simulated Pre-Limit Density, a=1.9

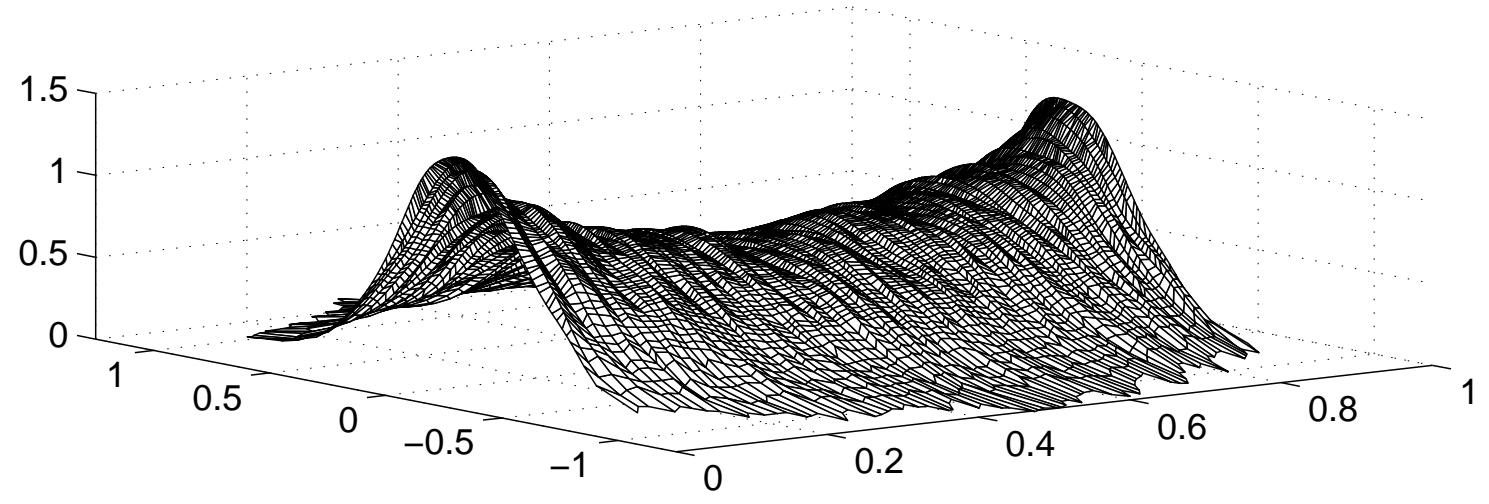

Simulated Limit Density, a=1.9

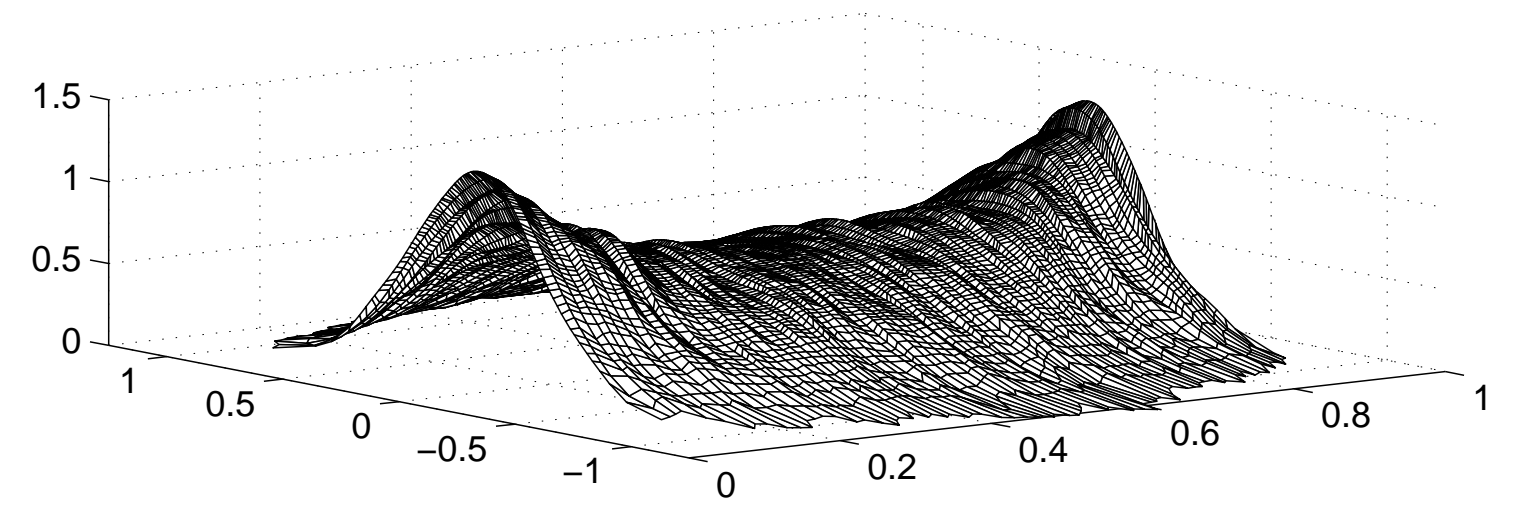


Figure 4. Simulated and Response-surface Fits of Critical Values

Simulated .90 Quantiles
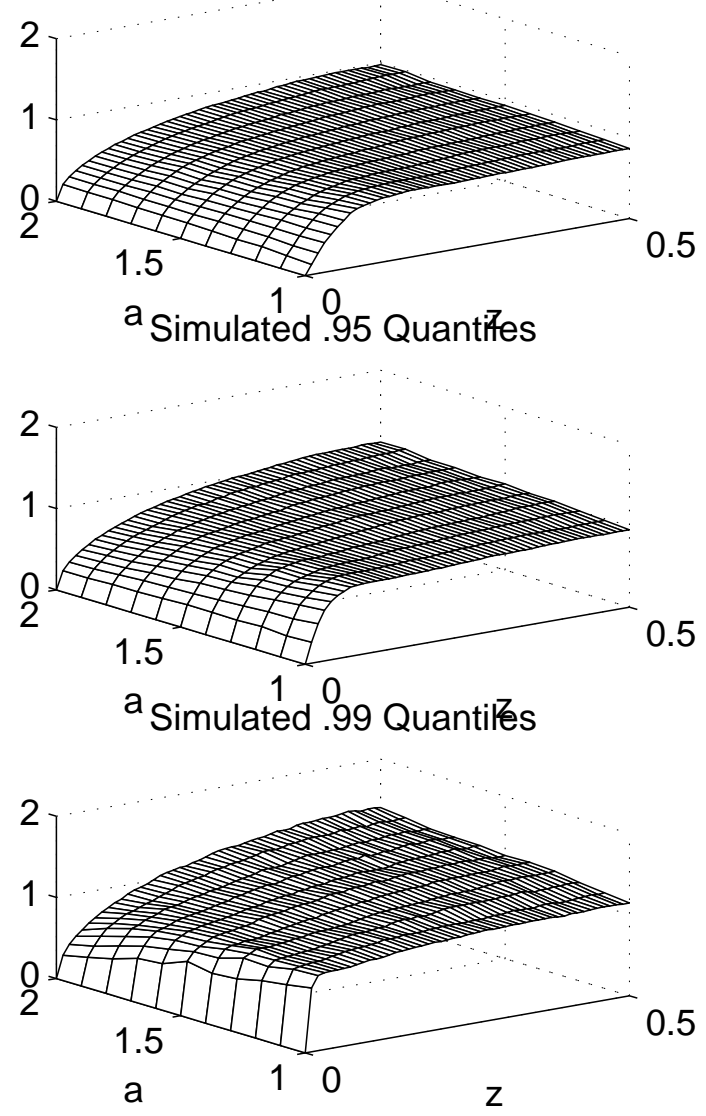

Fitted .90 Quantiles
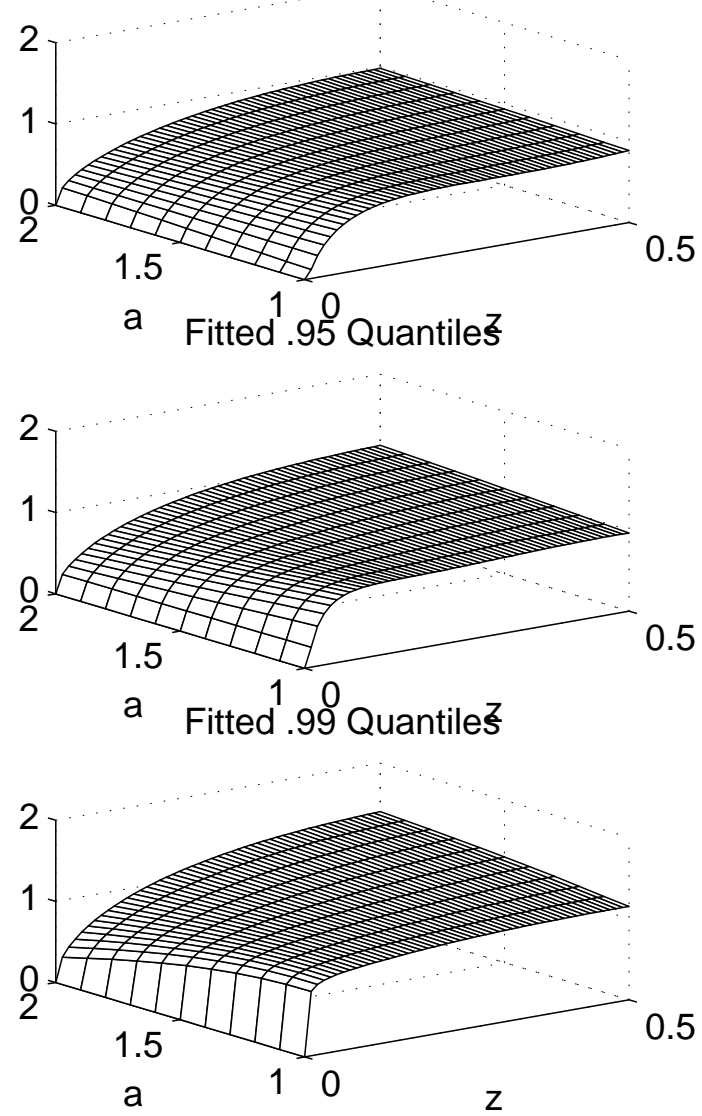